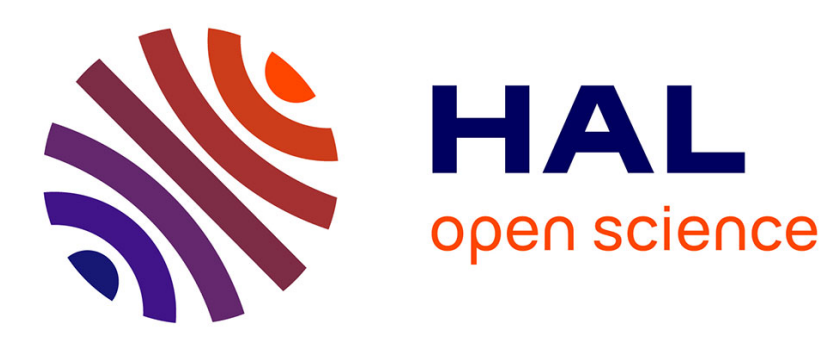

\title{
Subaqueous Barchan dunes in turbulent shear flow. Part 2: Fluid flow.
}

François Charru, Erick M. Franklin

\section{To cite this version:}

François Charru, Erick M. Franklin. Subaqueous Barchan dunes in turbulent shear flow. Part 2: Fluid

flow.. Journal of Fluid Mechanics, 2012, vol. 692, pp. 131-154. 10.1017/jfm.2011.528 . hal-00920714

\section{HAL Id: hal-00920714 \\ https://hal.science/hal-00920714}

Submitted on 19 Dec 2013

HAL is a multi-disciplinary open access archive for the deposit and dissemination of scientific research documents, whether they are published or not. The documents may come from teaching and research institutions in France or abroad, or from public or private research centers.
L'archive ouverte pluridisciplinaire HAL, est destinée au dépôt et à la diffusion de documents scientifiques de niveau recherche, publiés ou non, émanant des établissements d'enseignement et de recherche français ou étrangers, des laboratoires publics ou privés. 


\section{OATAO \\ Open Archive Toulouse Archive Ouverte}

\section{Open Archive TOULOUSE Archive Ouverte (OATAO)}

OATAO is an open access repository that collects the work of Toulouse researchers and makes it freely available over the web where possible.

This is an author-deposited version published in : http://oatao.univ-toulouse.fr/ Eprints ID : 10562

To link to this article : DOI:10.1017/jfm.2011.528

URL : http://dx.doi.org/10.1017/jfm.2011.528

To cite this version : Charru, François and Franklin, Erick M.

Subaqueous Barchan dunes in turbulent shear flow. Part 2: Fluid flow. (2012) Journal of Fluid Mechanics, vol. 692 . pp. 131-154. ISSN 14697645

Any correspondance concerning this service should be sent to the repository administrator: staff-oatao@ listes-diff.inp-toulouse.fr 


\title{
Subaqueous barchan dunes in turbulent shear flow. Part 2. Fluid flow
}

\author{
F. Charru† and E. M. Franklin \\ Université de Toulouse - Institut de Mécanique des Fluides de Toulouse - CNRS, Allée C. Soula, \\ 31400 Toulouse, France
}

We report an experimental study of the turbulent flow above a barchan dune in a channel, from particle image velocimetry measurements, for Reynolds numbers ranging from 9000 , just below the threshold for particle motion, up to 24000 , where the dune moves. Two calculations of the speed-up over the dune are compared, the usual 'same-elevation' and the more relevant 'Lagrangian', showing that the latter is smaller by a factor of two. The two-layer structure of the flow disturbance - an essentially inviscid outer layer and a turbulent inner layer of thickness $\delta_{i}$ - is assessed. In the outer layer, streamline curvature is shown to be responsible for half of the Lagrangian speed-up, from the comparison of the velocity measurements with two Bernoulli calculations. In the inner layer, detailed measurements of the velocity and stresses are provided, down to $y^{+} \approx 1$, and the momentum budget is discussed. The Reynolds shear stress decreases monotonically towards the dune surface, according to the standard mixing-length closure, whereas the total shear stress increases strongly in the viscous sublayer. Along the dune surface, the shear stress increases up to the crest where it reaches twice its unperturbed value. A good estimate of the surface stress is provided by a parabolic fit of the inner velocity profile matching the outer flow at $y_{d} \approx \delta_{i}$. Doubling the Reynolds number, the surface shear stress and the speed-up decrease by $\sim 30 \%$. The implications of these results on the dune motion, presented in Part 1 of this study (Franklin \& Charru, J. Fluid Mech., vol. 675, 2011, pp. 199-222), are finally discussed.

Key words: particle/fluid flow, sediment transport, turbulent boundary layers

\section{Introduction}

\subsection{Aim of the paper}

The presence of a bump, hill or dune on a flat ground perturbs the boundary-layer flow and introduces new space and time scales. Such a situation, encountered in aerodynamics, meteorology, and hydraulic engineering, raises fundamental issues such as the distribution of velocity and stresses, drag, flow separation, and the dispersion and trapping of passive scalars or inertial particles. Many studies have been devoted to the analysis of the perturbed flow over hills and moving waves (Taylor, Mason \& Bradley 1987; Belcher \& Hunt 1998), or over erodible ground such as desert dunes (Livingstone, Wiggs \& Weaver 2007) or river dunes (Best 2005). Fruitful insights have 
been provided, in particular, by asymptotic analyses exploiting the layered structure of the flow, which are briefly reviewed below.

Another issue in the above analyses is sand or particle transport in pipes and channels, e.g. in petroleum engineering or the food industry, where the particles form an irregular wavy bed sheared by the fluid flow. This kind of closed flow has received less attention than large-scale open flows. It may differ in several respects, for instance: (i) the dune size is not small in comparison with the channel width or the pipe diameter so that confinement effects can be expected; (ii) the Reynolds number is generally smaller than in open flows, so that viscous effects may be of importance; (iii) the flow regime may change from smooth, over the channel wall, to rough, over the dune, which is the opposite situation to that encountered for desert dunes, where the sand roughness is smaller than that of the pebbly surrounding ground. In Part 1 of this study (Franklin \& Charru 2011), experiments were reported on the formation and migration of isolated dunes in a closed channel. In the present Part 2, the fluid flow above these dunes is investigated, with particular emphasis on the assessment of the layered structure, stresses at the dune surface, and Reynolds number effects.

The paper is organized as follows. The theoretical background and available experimental and numerical results are first presented. The experimental apparatus used in the present study is briefly described in $\S 2$, along with some preliminary observations. The mean flow is analysed in $\S 3$ and the relevance of the distinction between two layers is discussed. Stresses are studied in $\S 4$, with particular emphasis on the shear stress at the dune surface. Reynolds number effects are investigated in $\S 5$, and a final discussion is given in the last section.

\subsection{Background}

Asymptotic analyses of the perturbed flow over a bump, of characteristic length $L$ and height $H$, are essentially based on the governing equations linearized for small slope $H / L$. Within this frame, the basic problem is that of the flow over a sinusoidal bottom. Although such analyses cannot account quantitatively for flow separation on the leeward side of steep bumps, a question that requires other methods (Scheichl, Kluwick \& Smith 2011), they have proved their efficiency for gentle slopes.

The laminar flow problem was first solved by Benjamin (1959), with particular emphasis on the stresses on the (possibly moving) bottom, in the context of the generation of water waves by the wind. Benjamin's analysis accounts for the common situation where the wave amplitude is not small compared to the characteristic length of variation of the velocity in the transverse direction, thanks to the use of an orthogonal curvilinear system of coordinates. The equations governing the twodimensional flow disturbance above a sinusoidal bottom with wavenumber $k$ then reduce to the steady Orr-Sommerfeld equation, for base flow $U_{0}(y)$ and viscosity $v$. Far from the wall, where viscosity effects are negligible, the Orr-Sommerfeld equation reduces to the Rayleigh equation, whose solution gives the pressure distribution close to the wall. Within a 'wall friction layer' of thickness $\delta_{i}=\left(v / k U_{0}^{\prime}(0)\right)^{1 / 3}$, thin compared to the wavelength and corresponding to the penetration depth of vorticity disturbances, viscous effects must be taken into account while the curvature of the base velocity profile can be neglected. Benjamin considered several unperturbed base flows, including the logarithmic mean velocity profile of a turbulent boundary layer, and showed in particular that the phase of the shear stress, a quantity of major importance for an erodible bottom, always leads that of a wavy wall.

The analysis of Jackson \& Hunt (1975) extends that of Benjamin (1959) to turbulent flow, typically for the logarithmic unperturbed velocity profile, $U_{0}=\left(u_{*} / \kappa\right) \ln \left(y / y_{0}\right)$ 
where $y_{0}$ is the hydrodynamic roughness. The main idea, similar to that of Benjamin, is that, for large $\ln \left(L / y_{0}\right)$ (in addition to small $H / L$ ), the flow perturbation over the bump has a two-layer structure: an inviscid outer layer, and an inner layer governed by the boundary layer equations (with the hydrodynamic roughness inherited from a third surface layer, with thickness of the order of the size of the roughness elements or the viscous length). In the inner layer, turbulence is expected to be at equilibrium so that the Reynolds shear stress may be modelled using the mixing length theory. The thickness $\delta_{i}$ of the inner layer can be derived from the balance of the longitudinal acceleration and the stress gradient; for logarithmic unperturbed velocity profile, it is given by the equation

$$
\left(\delta_{i} / L\right) \ln \left(\delta_{i} / y_{0}\right)=2 \kappa^{2} .
$$

(A slightly different expression, involving the square of the logarithm, has been proposed, and leads to lower $\delta_{i}$ : see Taylor et al. 1987.) The asymptotic theory of Jackson \& Hunt was improved and generalized in several directions, notably by Sykes (1980), who proposed a more rigorous expansion procedure in the small parameter $\left(u_{*} / U_{0}\right)^{1 / 2}$ and showed that, in the inner layer, Reynolds stresses are not in equilibrium except in a very thin surface layer. Other improvements include three-dimensional analysis (Mason \& Sykes 1979), a third intermediate layer accounting for vorticity in the outer flow (Hunt, Leibovich \& Richards 1988), stratified flow (Weng 1997), or flow over hills covered with a plant canopy (see Finnigan \& Belcher 2004). Scaling laws have been proposed for the maximum velocity perturbation (located above the summit of the hill, typically at the height $\left.\delta_{i} / 3\right)$, and the surface shear stress (proportional at leading order to the pressure perturbation imposed by the outer inviscid flow). The expression for the bottom shear stress derived by Hunt et al. (1988) (see the Appendix) is widely used in models for aeolian sand dunes (Weng et al. 1991; Andreotti, Claudin \& Douady 2002; Kroy, Sauermann \& Herrmann 2002; Sauermann et al. 2003).

Field observations and wind tunnel experiments have validated the main results of asymptotic analyses, at least for the mean flow predictions and gentle slopes: see the reviews by Taylor et al. (1987) and Belcher \& Hunt (1998). It was clear that the surface shear stress cannot be estimated confidently from one single velocity measurement and the assumption of logarithmic profile (Weng et al. 1991; Wiggs, Livingstone \& Warren 1996; Livingstone et al. 2007), and that shear stress models developed for engineering purposes (Smith \& McLean 1977) are of limited use: see the discussion by Kostaschuk, Villard \& Best (2004). Wind tunnel experiments confirmed the inviscid dynamics of the perturbation in the outer flow, and showed maximum speed-ups in reasonable agreement with theoretical predictions (Britter, Hunt \& Richards 1981; Gong \& Ibbetson 1989; Finnigan et al. 1990; Athanassiadou $\&$ Castro 2001). Wind tunnel experiments on model hills were shown to reproduce the speed-ups measured on full-scale, three-dimensional low hills, with little effect from the hydrodynamic roughness (Teunissen et al. 1987). As for the Reynolds stresses, they increase above the upstream foot of the hill, then decrease towards the hill crest, and increase again in the wake region. Although measurements display some scatter, they are, in the outer layer, in qualitative agreement with predictions of the rapid distortion theory (Britter et al. 1981; Zeman \& Jensen 1987; Finnigan et al. 1990; Ross et al. 2004). In the inner layer, the usual mixing-length modelling of the shear stress has been questioned (Sykes 1980), but provides reasonable predictions for the mean flow (Poggi et al. 2007); however, more elaborated closure schemes are necessary for good shear stress predictions (Ross et al. 2004). Streamline curvature has 


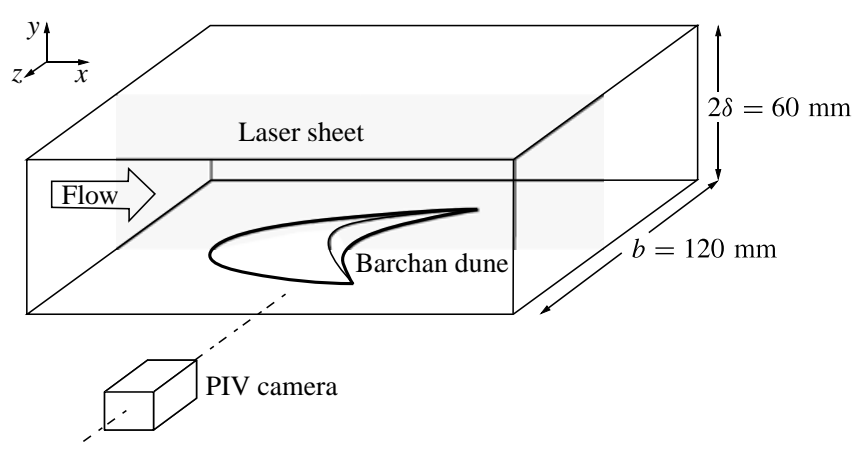

FIGURE 1. Sketch of the experimental set-up and a barchan dune.

been recognized to have a strong effect on the structure of the turbulence: concave streamlines (above the upwind foot of the hill) promote increased turbulent mixing whereas convex streamlines (over the crest) damp turbulence (Zeman \& Jensen 1987; Finnigan et al. 1990), with important consequences on sand transport for erodible dunes (Weng et al. 1991; Wiggs et al. 1996; van Boxel, Arens \& van Dijk 1999; Walker \& Nickling 2003).

Numerical simulation allowed various turbulence closures to be compared (Abrams \& Hanratty 1985; Ross et al. 2004), while large eddy simulation reproduces the mean flow features and provides reasonable predictions for the stresses and the detached and secondary flows (Gong, Taylor \& Dörnbrack 1996; De Angelis, Lombardi \& Banerjee 1997; Henn \& Sykes 1999; Yue, Lin \& Patel 2006). In particular, Abrams \& Hanratty (1985) showed that for smooth wavy walls (with wavenumber $k v / u_{*}>10^{-4}$, and maximum slope $=0.044$ so that nonlinear effects are expected to be weak), usual turbulence models assuming local equilibrium fail to reproduce the phase angle of the shear stress, whereas introducing some longitudinal relaxation of the Reynolds stresses (Sykes 1980) improves the predictions. Although the relevance of turbulence models based on mixing lengths is still debated, such models are widely used for the study of the instability of an erodible bed (Richards 1980; Sumer \& Bakioglu 1984; Fourrière, Claudin \& Andreotti 2010; Colombini \& Stocchino 2011). Finally, nonlinear effects related to finite slope have been taken into account either heuristically or from Landau-Stuart expansions (Belcher \& Hunt 1998; Colombini \& Stocchino 2011); such effects may be involved in the observed discrepancies between linear predictions and measurements, for maximum wave slope larger than, say, 0.15 (Fourrière et al. 2010).

As shown below, the conditions of small $H / L$ and large $\ln \left(L / y_{0}\right)$ relevant to linear asymptotic analyses are fulfilled for the small-scale barchan dunes investigated here.

\section{Experimental apparatus and first observations}

\subsection{Apparatus}

The experimental apparatus is the same as that presented in Franklin \& Charru (2011), so that its description is only briefly sketched here. It mainly consists of a horizontal Plexiglas channel, $6 \mathrm{~m}$ long, with rectangular cross-section of height $2 \delta=60 \mathrm{~mm}$ and width $b=120 \mathrm{~mm}$ (figure 1). Small conical heaps of particles were formed in the channel by dropping the particles from a small hole in the upper wall located at $4.15 \mathrm{~m}$ from the entrance of the channel. Then the flow was started up, and the heap quickly deformed into a barchan dune. 
The bulk velocity $U_{b}$ of the flow, defined as the ratio of the measured volumetric flow rate and the channel cross-section, was varied between 0.15 and $0.4 \mathrm{~m} \mathrm{~s}^{-1}$. The corresponding Reynolds number

$$
R e=\frac{U_{b} 2 \delta}{v}
$$

was in the range 9000-24000. As shown in Part 1, the water flow has, in the last third of the channel, the classical features of a fully developed turbulent channel flow. The mean flow velocity $U_{0}(y)$ is well represented by the classical log-law, $U_{0}^{+}=(1 / \kappa) \ln y^{+}+B$ in wall units, for $y^{+}$in the range 30-200 $(2 \lesssim y(\mathrm{~mm}) \lesssim 10)$, with the von Kármán coefficient $\kappa=0.41$ and the additive constant $B=5.5$ having their usual values (Davidson 2004). The shear velocity $u_{*}$, as determined by curve fitting, was found to agree with that predicted by the Blasius correlation

$$
c_{f}=\frac{u_{*}^{2}}{\frac{1}{2} U_{b}^{2}}=0.079(1.33 R e)^{-1 / 4},
$$

where $(1.33 R e)$ is the Reynolds number based on the hydraulic diameter of the rectangular channel. The whole velocity profile, from the lower wall $(y=0)$ up to the centreline $(y=\delta)$, was found to be nicely fitted by adding to the log-law two usual corrections: Spalding's correction near the wall and the Coles-Lewkowicz law of the wake in the centre part of the channel (Panton 2007). In particular, the measurements and the above fit gave nearly the same maximum velocity at the centreline, $U_{\delta}=U_{0}(\delta) \approx 1.19 U_{b}$, with maximum difference less than $2 \%$ in the explored range of Reynolds number. Reynolds stresses were also shown to have their usual values, with nearly constant shear stress $-\overline{u^{\prime} v^{\prime}}$, close to $u_{*}^{2}$, in the region $30<y^{+}<200$ where the log-law holds.

\subsection{Velocity measurements above the dune}

For the mean flow to be close to stationary in the fixed reference frame of the camera, the dune velocity had to be as small as possible, which led us to choose heavy zircon particles with median diameter $0.19 \mathrm{~mm}$ and density $\rho_{p}=3760 \mathrm{~kg} \mathrm{~m}^{-3}$ (those of Series 4 used in Part 1). The settling velocity of these particles is $V_{\text {fall }}=35 \mathrm{~mm} \mathrm{~s}^{-1}$, and their settling Reynolds number is $R e_{\text {fall }}=V_{\text {fall }} d / v=6.6$. For the largest fluid flow rate, the particle Reynolds number was $R e_{p}=u_{*} d / v=4.0$. The choice of the dune size had to meet two opposite requirements: it had to be sufficiently large to induce significant perturbations of the fluid flow and sufficiently small to keep the dune in the logarithmic region and ensure a small confinement effect of the upper and lateral walls. The corresponding length and height were typically 40 and $5 \mathrm{~mm}$, the latter corresponding to one-third, at most, of the thickness of the logarithmic layer.

Fluid velocity measurements were performed using particle image velocimetry (PIV) and images were processed with the software PIVIS developed at IMFT: see Part 1 for details (Franklin \& Charru 2011). Once a barchan dune had formed from the initial heap of particles, the laser sheet was positioned in the vertical symmetry plane of the dune (which might be slightly different from the symmetry plane of the channel), as shown in figure 1 . The field of view covered either the whole dune and channel height, or a smaller area for better spatial resolution. For most of the figures presented here, this field was $22 \mathrm{~mm} \times 17 \mathrm{~mm}$, corresponding to a spatial resolution of the velocity field of $0.14 \mathrm{~mm}$ in both directions (with correlation boxes of $16 \times 16$ pixels and overlap of $50 \%$ ). Due to smudges of glue at the corners of the Plexiglas channel, 

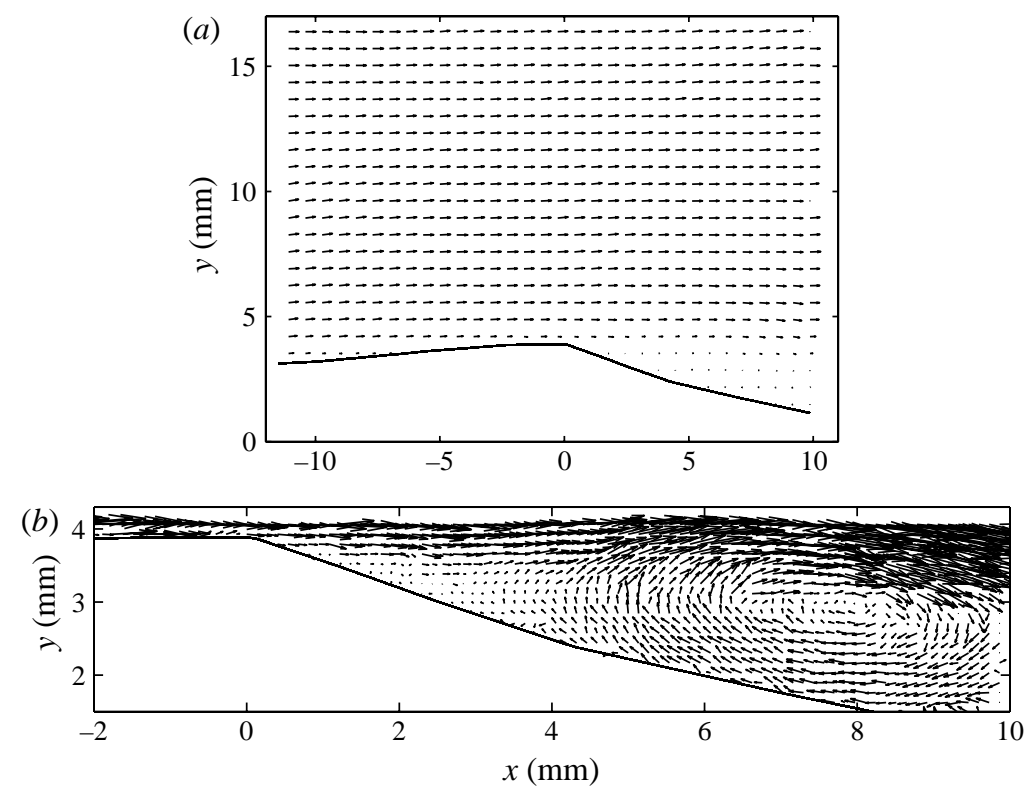

FIGURE 2. (a) Typical velocity field in the vertical symmetry plane above a dune (solid line). (b) Enlargement of the region downstream of the brink. $U_{\delta}=0.184 \mathrm{~m} \mathrm{~s}^{-1}\left(R e=9260, u_{*}=\right.$ $\left.9.45 \mathrm{~mm} \mathrm{~s}^{-1}\right)$.

the fluid flow close to the lower wall was blurred up to a distance of about one millimetre, leading there to uncertain measurements which will not be reported. The time interval between the two frames of a pair ranged from $224 \mu$ s (for the highest Reynolds number) to $496 \mu$ s (for the lower one), and the frequency of the pairs was $4 \mathrm{~Hz}$. The number of pairs per run ranged from 108 to 864, which was chosen such that the displacement of the dune was small during one run. This displacement was $3.5 \mathrm{~mm}$ for the highest Reynolds number and much less for the lower ones. The mean velocity and Reynolds stresses were obtained by averaging the instantaneous fields; convergence within $1 \%$ was achieved with a number of fields (i.e. the number of pairs of images) of $\sim 30$ for the mean velocity and 200 for the Reynolds stresses.

\subsection{Preliminary observations}

Figure 2(a) shows a typical instantaneous velocity field in the vertical symmetry plane of a dune, with $y=0$ corresponding to the bottom wall and $x=0$ to the position of the brink of the dune profile. The flow velocity $U_{b}=0.184 \mathrm{~m} \mathrm{~s}^{-1}(R e=9260)$ is just below the threshold for particle motion, so that the dune is at rest. An enlargement of the region downstream of the slip face is displayed in figure $2(b)$, showing the existence of a recirculating flow (note that the base of the slip face is hidden by the horn in between the laser sheet and the camera, so that fluid may flow across the apparent dune boundary). The recirculating flow is strongly unsteady: as soon as it is formed, a vortex detaches from the dune, another vortex then growing at the same place. However, the sampling frequency of the pairs of images was not high enough to provide a detailed picture of the vortex dynamics, in particular the distribution of the detachment times. In order to get a wider picture of the flow along the dune while keeping high spatial resolution, one or two more flow fields were recorded upstream 

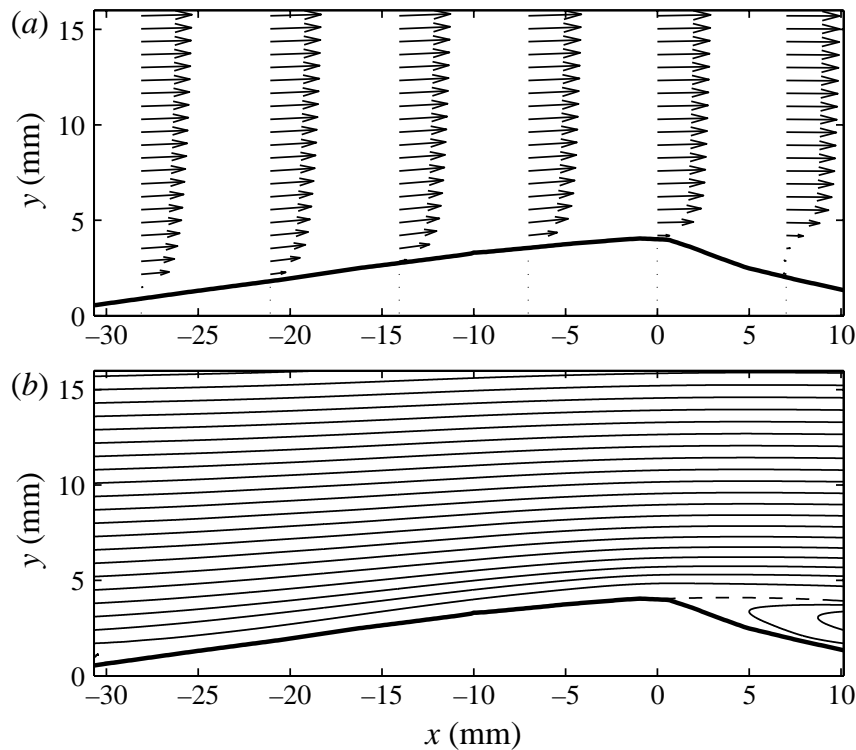

FIgURE 3. Typical mean flow above a dune, for $U_{\delta}=0.184 \mathrm{~m} \mathrm{~s}^{-1}\left(R e=9260, u_{*}=\right.$ $9.45 \mathrm{~mm} \mathrm{~s}^{-1}$ ): (a) vertical profiles of the velocity; $(b)$ streamlines. Dashed line, dune profile fitted by (2.3) with $h_{M}=4.1 \mathrm{~mm}, L=23 \mathrm{~mm}, x_{0}=4 \mathrm{~mm}$. The dune height at the brink is $H=4.0 \mathrm{~mm}$.

or downstream of that centred on the dune brink. Most of the figures shown in the following correspond to two or three adjacent flow fields merged together.

Figure 3(a) shows typical vertical profiles of the time-averaged velocity in the symmetry plane of a dune, at longitudinal positions equally spaced from the dune brink. Figure 3(b) displays the corresponding streamlines, which can be seen to come closer to each other above the dune crest, as expected, and reach their highest point slightly downstream of the brink. The detachment of the boundary layer downstream of the slip face and the mean back-flow in the wake are also clearly visible.

Figure $3(b)$ also displays a fit of the dune profile (dashed line) with the cosine square function

$$
h(x)=h_{M} \cos ^{2} \frac{\pi\left(x-x_{0}\right)}{4 L},
$$

where $L=23 \mathrm{~mm}$ is the half-length defined as the horizontal distance from the summit to the point where the height is $h_{M} / 2$. The cosine square function, frequently chosen for dune models (e.g. Gong \& Ibbetson 1989; Ross et al. 2004), appears to fit the dune over its entire profile (the fit and the dune profile are indistinguishable). The half-length $L$, different from the length used in Part 1 of this study, corresponds to that used in most previous studies and in the definition (1.1) of the inner layer thickness. An important point is that the dune brink does not coincide with the summit of the fit, which is located at the distance $x \approx 4 \mathrm{~mm} \approx h_{M}$ downstream. However, the height $H$ of the dune at the brink is only slightly smaller than $h_{M}$, typically by $0.1 \mathrm{~mm}$. The dune maximum slope, $\pi h_{M} / 4 L$, is $0.14\left(8^{\circ}\right)$. 
(a)

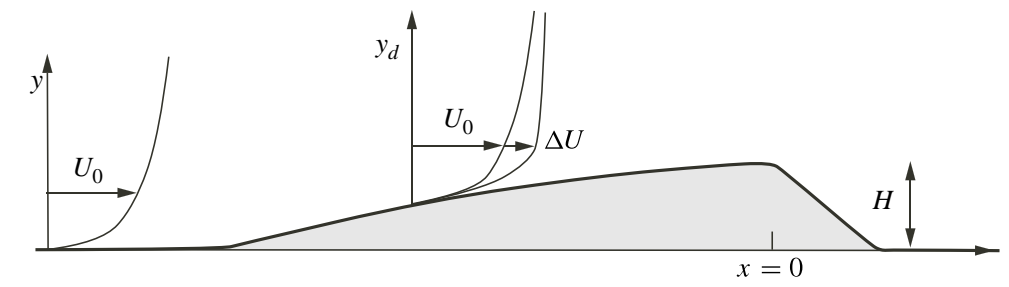

(b)

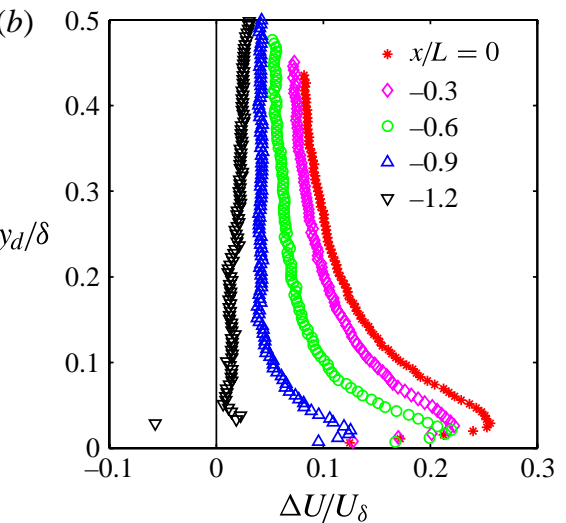

(c)

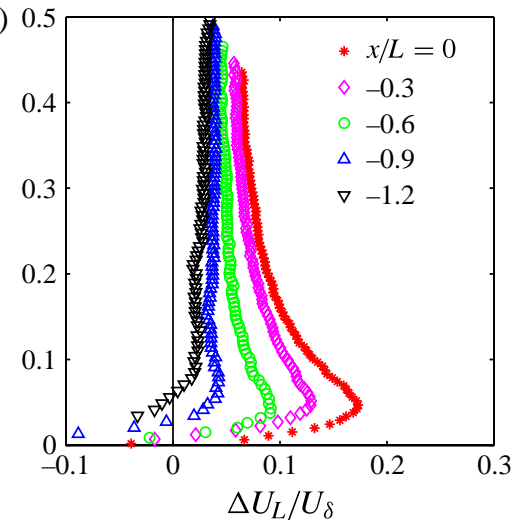

Figure 4. (a) Sketch of the definition of the speed-up $\Delta U$. (b) Profiles of the 'sameelevation' speed-up (3.1) at five $x$-positions upstream of the crest $(x / L=0) ;(c)$ profiles of the Lagrangian speed-up (3.2) at the same $x$-positions. Parameters: see figure 3.

\section{Mean flow}

\subsection{Mean velocity and speed-up}

From figure 3(a), the speed-up of the fluid flow near the dune crest can clearly be seen. When plotted with logarithmic vertical scale, the longitudinal velocity profiles do not exhibit any straight line (not shown), confirming another well-known feature that over a dune or hill, velocity profiles are not logarithmic. A deeper insight into the disturbed flow can be gained from the speed-up $\Delta U$ at the distance $y_{d}=y-h(x)$ above the dune,

$$
\Delta U(x, y)=U(x, y)-U_{0}\left(y_{d}\right),
$$

i.e. the difference between the actual velocity $U(x, y)$ and the unperturbed velocity $U_{0}\left(y_{d}\right)$ at the same elevation above the bottom wall far upstream of the dune (figure $4 a$ ). In the following, $U_{0}(y)$ is the logarithmic law with the wall and wake corrections included and the friction velocity taken from the Blasius correlation (2.2). Figure 4(b) displays vertical profiles of the speed-up $\Delta U$ at the dune crest $(x / L=0)$ and four upstream positions $(x / L<0)$, normalized with the maximum velocity $U_{\delta}=U_{0}(\delta)$. The speed-up profile at $x / L=-1.2$ appears to be nearly flat, but a peak develops as the dune crest is approached, at the height $y_{d} \approx 0.02 \delta$. The maximum of the peak is reached at the crest, with value $\Delta U \approx 0.26 U_{\delta} \approx 4.3 u_{*}$.

The above 'same-elevation' speed-up is the simplest to determine, but does not correspond to the actual variation of the Lagrangian velocity of a fluid particle. Along any streamline $y_{s}(x)$ (or trajectory) above the dune, this Lagrangian speed-up is

$$
\Delta U_{L}\left(x, y_{s}\right)=U\left(x, y_{s}\right)-U_{0}\left(y_{s 0}\right),
$$




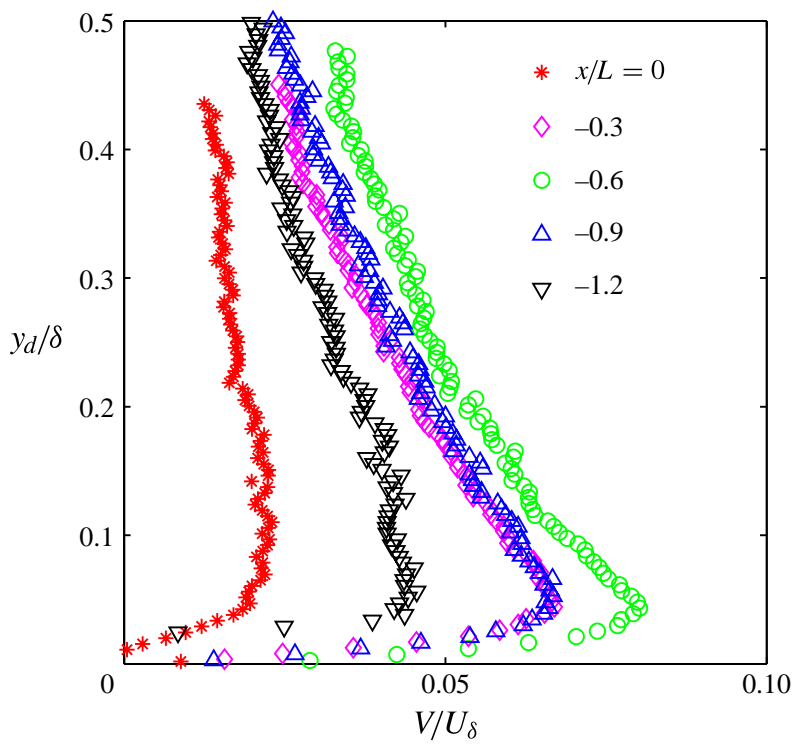

FIGURE 5. Profiles of the vertical velocity $V$, normalized with $U_{\delta}$, at five $x$-positions upstream of the crest $(x / L=0)$. Parameters: see figure 3 .

where $U\left(x, y_{s}\right)$ is the measured velocity and $U_{0}\left(y_{s 0}\right)$ is the unperturbed upstream velocity on the same streamline (with elevation $y_{s 0}$ above the wall). Since the spanwise velocity is zero in the symmetry plane of the dune, streamlines could be computed from the measured mean velocity field as the lines tangent everywhere to the plane vector field (as those shown in figure $3 b$ ). However, the field of view did not extend far enough upstream for the location $y_{s 0}$ of the undisturbed streamlines to be determined accurately, so that $y_{s 0}$ had to be estimated from some extrapolation. This was done by assuming plane flow upstream of the field of view (i.e. negligible divergence of the streamlines in the horizontal plane), allowing $y_{s 0}$ to be calculated from conservation of the flow rate

$$
\int_{0}^{y_{s 0}} U_{0}(y) \mathrm{d} y=\int_{h\left(x_{u}\right)}^{y_{s}} U\left(x_{u}, y\right) \mathrm{d} y,
$$

where $x_{u}$ is the upstream boundary of the field of view of the camera.

Vertical profiles of the Lagrangian speed-up are displayed in figure 4(c); they are similar to those of figure $4(b)$ but with two differences. Close to the crest $(x / L \gtrsim-0.6)$, the speed-up is smaller by a factor of about two (because the compression of the streamlines is now accounted for), and is reached at a higher elevation above the dune, $y_{d} \approx 0.05 \delta$. Further upstream $(x / L \lesssim-0.9)$, there is no peak and the speed-up is negative near the dune surface, because of the vertical divergence of the streamlines. (Note that the relative speed-up, i.e. the Lagrangian speed-up normalized with the unperturbed velocity $U_{0}\left(y_{d}\right)$, exhibits larger values (not shown) and reaches $40 \%$ at the crest.)

Profiles of the vertical velocity $V\left(x, y_{d}\right)$ are shown in figure 5 . These profiles all exhibit a peak at the elevation $y_{d} \approx 0.05 \delta$, above which $V$ slowly decreases. At a given elevation above the dune, $V$ first increases and then decreases as the crest is approached, with largest velocity at the position $x / L \approx-0.6$. 

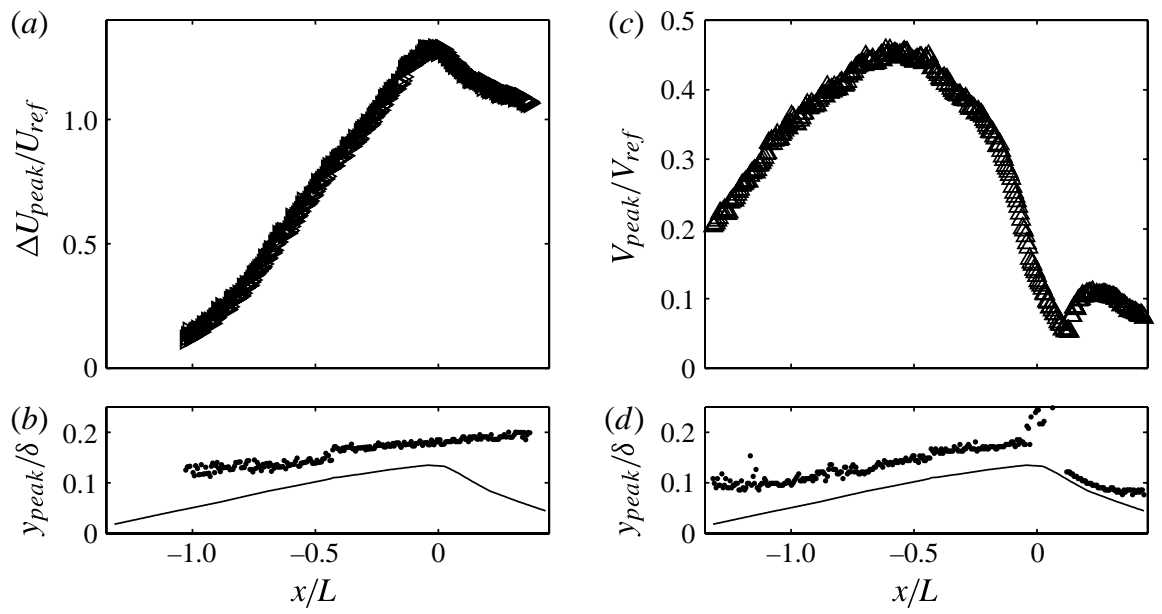

FIGURE 6. (a) Variation along the dune of the peak of the speed-up, $\Delta U_{L \text {,peak }}$, normalized with $U_{r e f}=(H / \delta) U_{\delta}$. (b) Vertical position of $\Delta U_{L, p e a k}$ (dots) and the dune profile for reference. $(c, d)$ The same for the peak of the vertical velocity, $V_{\text {peak }}$, normalized with $V_{\text {ref }}=(H / L) U_{\delta}$. Parameters: see figure 3 .

The variations along the dune of the peaks of the Lagrangian speed-up, $\Delta U_{L, p e a k}$, and vertical velocity, $V_{\text {peak }}$, as well as their vertical locations, are plotted in figure 6. It appears that $\Delta U_{L, p e a k}$ increases linearly up to the dune crest and then decreases (figure $6 a$ ), with a maximum of $1.3 U_{\text {ref }}$ where $U_{\text {ref }}=(H / \delta) U_{\delta}$ is the expected order of magnitude; the distance of the peak from the dune surface slightly decreases from $0.06 \delta$ at $x / L=-1-0.05 \delta$ at the crest $(x / L=0)$ (figure $6 b$ ). The corresponding variations of $V_{\text {peak }}$ are displayed in figures $6(c)$ and $6(d)$. The peak value increases and then decreases, with a maximum of $0.47 V_{\text {ref }}$ where $V_{\text {ref }}=(H / L) U_{\delta}$ is the expected order of magnitude; this maximum is reached at $x / L \approx-0.6$ close to the location of the inflexion point of the streamlines. The secondary peak downstream of the crest (at $x / L \approx 0.2)$ is related to the vortex in the wake of the dune.

\subsection{Analysis of the flow in the outer layer}

As discussed in the Introduction, the flow disturbance over a dune or hill can be analysed, for large $U_{0} / u_{*}$ and $\ln \left(L / y_{0}\right)$, as the superposition of two layers: an inviscid outer layer, and an inner layer where friction is significant and the flow is driven by the pressure gradient inherited from the outer layer. The maximum slope of the dune profile is 0.14 (see $\S 2.3$ ), smaller than the usual limit of 0.15 for linear analyses to be accurate (see $\S 1.2$ ). Moreover, the parameters $U_{0} / u_{*}=19.5$ and $\ln \left(L / y_{0}\right)=7.6$ are reasonably large, so that the asymptotic analysis should be relevant. Then, the thickness $\delta_{i}$ of the inner layer can be estimated from several arguments (Belcher \& Hunt 1998), all of them giving here nearly the same value. Taking $L=23 \mathrm{~mm}$ and $y_{0}=d / 30$, where $d=0.2 \mathrm{~mm}$ is the grain diameter, the relation (1.1) gives $\delta_{i}=1.5 \mathrm{~mm}$. Since the grain roughness is small $\left(d^{+}=1.9\right.$ for $\left.u_{*}=9.45 \mathrm{~mm} \mathrm{~s}^{-1}\right)$, the velocity profile over a smooth wall may be preferred for $U_{0}$, which gives the close result $\delta_{i}=1.6 \mathrm{~mm}$. A simpler estimation of $\delta_{i}$ arises from the balance of the horizontal advection time $L / U_{0}\left(\delta_{i}\right)$ and the vertical diffusion time $\delta_{i} / u_{*}$, which gives $\delta_{i}=1.9 \mathrm{~mm}$. Among the three values above, the intermediate value $\delta_{i}=1.6 \mathrm{~mm}$ is retained in the following (for which we note that $U_{0}\left(\delta_{i}\right) \approx 0.56 U_{\delta}$ ). Thus, the peaks of $\Delta U_{L}$ and $V$ lie 


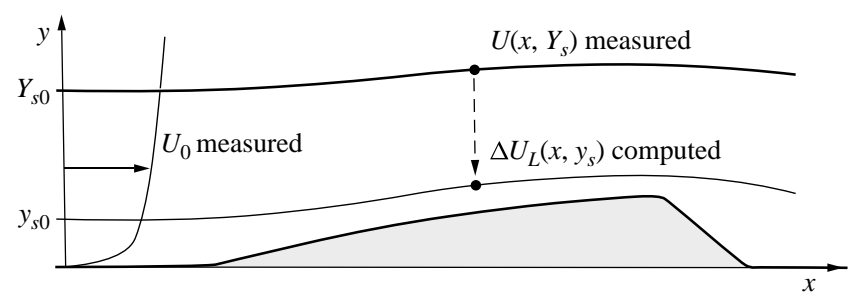

FIGURE 7. Sketch of the determination of the velocity $U\left(x, y_{s}\right)$ in the outer flow, from the undisturbed velocity profile $U_{0}(y)$ and the measured velocity $U\left(x, Y_{s}\right)$ along a single streamline $y=Y_{s}(x)$ far from the dune.

at $y_{d} \approx 0.05 \delta \approx 0.94 \delta_{i}$, very close to the boundary $y_{d}=\delta_{i}$ (see figure 6 ). Also, since $\delta_{i}=1.6 \mathrm{~mm}$ corresponds to $\delta_{i}^{+}=15$ in wall units, viscous effects are expected to play a significant role in the inner layer.

The existence of an inviscid outer layer $\left(y_{d}>\delta_{i}\right)$ is assessed here, not by using the expressions provided by Hunt et al. (1988), but in another way accounting for the geometrical complications of the present flow, i.e. flow height and width not large compared to the dune size $(\delta / H=7$ and channel width/dune width $=2)$, while avoiding its complete calculation. The starting point is to consider that the properties of the perturbed flow are embedded in the variations of the velocity along any particular streamline. Choosing the topmost streamline in the field of view of the camera, given by, say, $y=Y_{s}(x)$ with $Y_{s} / \delta \approx 0.5$ (see figure 7 ), the pressure perturbation along this streamline is given by the Bernoulli relationship

$$
\Delta p_{B}\left(x, Y_{s}\right)=\frac{1}{2} \rho\left(U_{0}^{2}\left(Y_{s 0}\right)-U^{2}\left(x, Y_{s}\right)-V^{2}\left(x, Y_{s}\right)\right),
$$

where $Y_{s 0}$ is the height of the undisturbed streamline upstream of the dune and $U$ and $V$ are the measured velocities. The pressure perturbation $\Delta p_{B}$ and velocity $U_{B}$ along any streamline $y=y_{s}(x)$ are then solutions of the inviscid momentum equations

$$
\begin{gathered}
\Delta p_{B}\left(x, y_{s}\right)=\frac{1}{2} \rho\left(U_{0}^{2}\left(y_{s 0}\right)-U_{B}^{2}\left(x, y_{s}\right)\right), \\
\Delta p_{B}\left(x, y_{s}\right)-\Delta p_{B}\left(x, Y_{s}\right)=-\int_{y_{s}}^{Y_{S}} \rho \frac{U_{B}^{2}}{R} \mathrm{~d} y,
\end{gathered}
$$

where $R^{-1}=y_{s}^{\prime \prime}\left(1+y_{s}^{\prime 2}\right)^{-3 / 2}$ is the curvature of the streamline. Taking the streamlines as determined from the velocity measurements, the above equations can be used to determine the pressure perturbation $\Delta p_{B}$ and the Lagrangian speed-up, as sketched in figure 7. It has been verified that, due to the small slope of the streamlines, the second-order contribution of the normal velocity $V$ is negligible in (3.5) and can be omitted, and that integration normal to the streamlines can be safely replaced by integration along the $y$-direction. Note that the above procedure cannot be considered as a complete calculation of the perturbed flow since it involves knowledge of the streamlines of the total flow, but it allows the assumption of inviscid flow to be assessed.

The resulting prediction of the speed-up is shown in figure 8, along with the measurements already shown in figure $4(c)$. It can be seen that in the outer layer (above the dash-dotted line corresponding to $\delta_{i}=1.6 \mathrm{~mm}$ ), the inviscid calculation (thick lines) matches the experiments quite well. Figure 8 also displays the predicted speed-up when the streamline curvature is ignored, i.e. $R=\infty$ in $(3.5 b)$ or $\partial_{y} p=0$ 


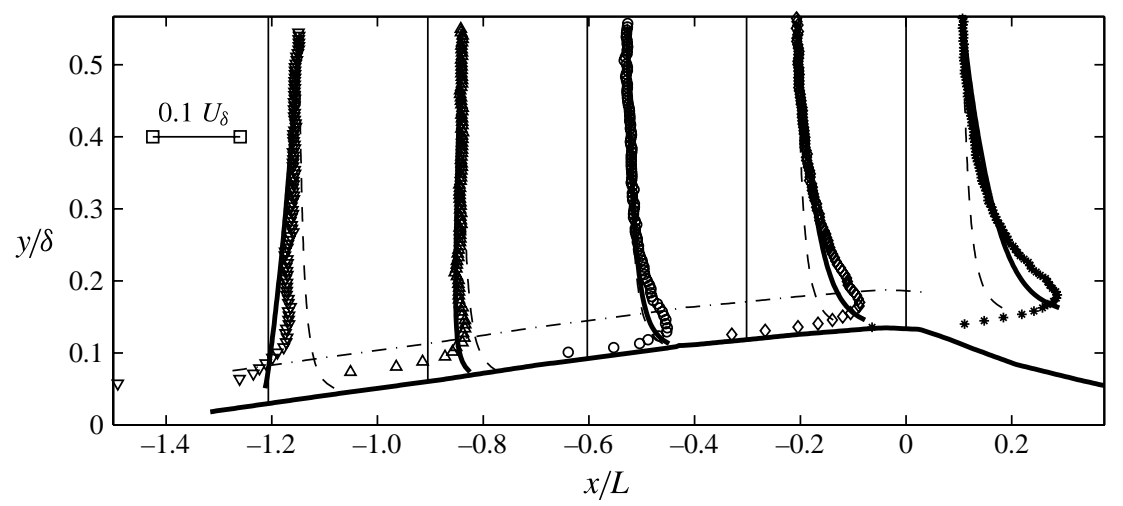

FIGURE 8. Vertical profiles of the Lagrangian speed-up $\Delta U_{L}$ over the dune: markers, experiments; - , inviscid calculation $(3.5)$; - - , streamline curvature ignored; $-\cdots$, lower limit of the outer layer at $y_{d}=\delta_{i}=1.6 \mathrm{~mm}$. Parameters: see figure 3 .

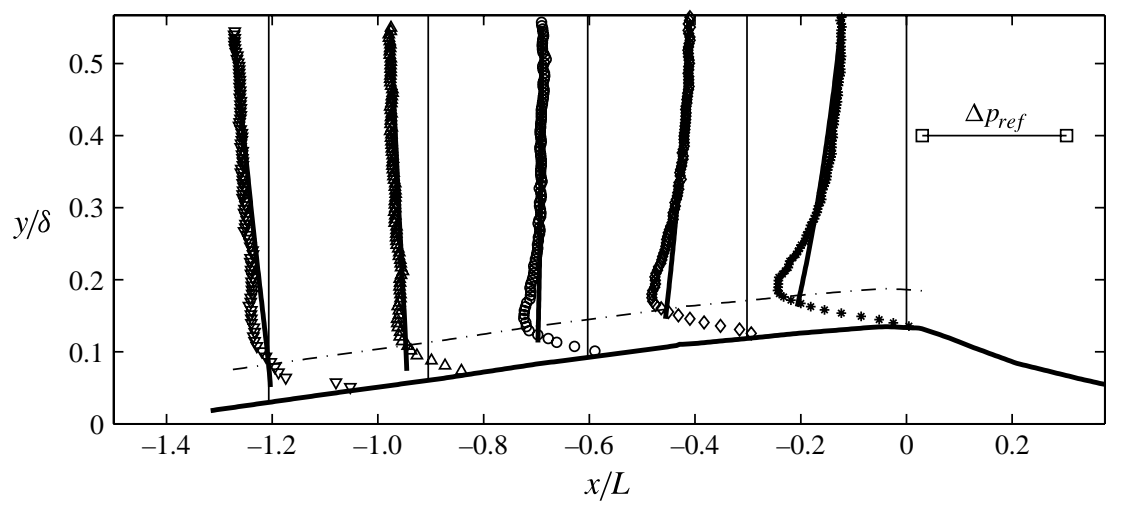

FIGURE 9. Vertical profiles of the pressure perturbation $\Delta p$ over the dune: markers, experiments; - , inviscid calculation $(3.5 a)-(3.5 b) ;-\cdot-$, lower limit of the outer layer with $\delta_{i}=1.6 \mathrm{~mm} . \Delta p_{\text {ref }}=\rho U_{\delta}^{2}(H / \delta) \approx 4.6 \mathrm{~Pa}$. Parameters: see figure 3 .

(dashed lines). As expected, this calculation overpredicts the speed-up near the foot of the dune, since it ignores the retarding effect of the positive curvature associated with larger pressure; conversely, it underpredicts the speed-up near the crest since it ignores the acceleration due to the negative curvature and lower pressure.

The above analysis is confirmed by the pressure profiles shown in figure 9, which shows good correspondence between the pressure perturbation predicted from (3.5) and the measured decrease of kinetic energy $(1 / 2) \rho\left(U_{0}^{2}-U^{2}\right)$. It appears that the Bernoulli decrease of the pressure far above the dune $(y / \delta \approx 0.5$, where streamline curvature is small) is counteracted by the centrifugal effect near the foot of the dune, and enhanced near the crest. Both effects are of the same order of magnitude: the magnitude of the Bernoulli decrease is $\Delta p_{\text {ref }}=\rho U_{\delta}^{2}(H / \delta) \approx 4.6 \mathrm{~Pa}$, while that of centrifugal effect is $\rho U_{\delta}^{2}(\delta / R) \approx 9.7 \mathrm{~Pa}$ (with the streamline curvature $R^{-1}$ estimated from that of the dune profile, $\pi^{2} H / 8 L^{2}$ according to (2.3)). 


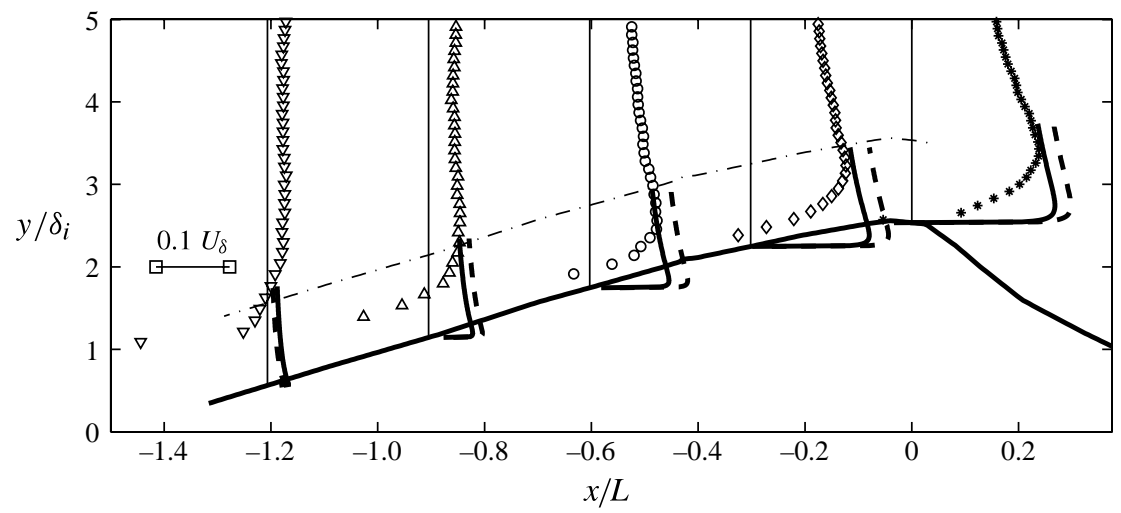

FIGURE 10. Vertical profiles of the measured speed-up $\Delta U_{L}$ (markers), and comparison with the inner layer calculations by Hunt et al. (1988): - , bell-shaped dune $h_{M} /\left(1+(x / L)^{2}\right)$; - Gaussian dune $\left.h_{M} \exp \left(-(x / L)^{2}\right)\right) ;-\cdot-$, upper boundary of the inner layer with $\delta_{i}=1.6 \mathrm{~mm}$. Parameters: see figure 3 .

\subsection{Analysis in the inner layer}

In the inner layer $\left(y_{d} \lesssim \delta_{i}=1.6 \mathrm{~mm}\right)$, the vertical profiles of the speed-up exhibit a peak below which the speed-up decreases to zero at the dune surface (see figure 8). In this layer, Reynolds stresses are no longer negligible, and measurements may be compared with the predictions of Hunt et al. (1988). From their analysis, the flows over two symmetric dune profiles have been computed: the bell profile $h_{M} /\left(1+(x / L)^{2}\right)$ and the Gaussian profile $h_{M} \exp \left(-(x / L)^{2}\right)$ (see the Appendix for details). The resulting speed-up is shown in figure 10. It can be seen that the agreement with the measurements is poor, for both dune profiles: although the value of the peak is not far from that measured, the peak is located very close to the dune surface, where the speed-up consequently exhibits very large gradient.

The failure of the above predictions is due to viscous effects, since the thickness of the inner layer, in wall units, is only $\delta_{i}^{+}=\delta_{i} u_{*} / v=15$. This is confirmed by the profiles of the velocity gradient $U^{\prime}(y)$ shown in figure 11 , at the same five longitudinal locations as before (and normalized with $u_{*}^{2} / v$ so that a velocity gradient equal to unity at the dune surface corresponds to the unperturbed shear stress $\rho u_{*}^{2}$ on the smooth channel wall). All profiles are similar and show that, for $y_{d}^{+} \lesssim 8$, the velocity gradient increases approximately linearly towards the dune surface, as for viscous Poiseuille flow. The value reached at the dune surface is about twice that at the smooth wall; this point is discussed further in the next section, devoted to stresses.

The linear variation of the velocity gradient suggests a parabolic fit of the velocity profiles, of the form $a y_{d}^{2}+b y_{d}$ (the following development was inspired by the Stratford method for the determination of the separation point in a boundary layer: see Schlichting \& Gesten 2000). The constants $a$ and $b$ have been chosen so that the parabola smoothly joins the outer flow $U_{B}\left(x, y_{s}\right)$ along a particular streamline $y_{s}(x)$ close to $y_{d}=\delta_{i}$, that is,

$$
a y_{s}^{2}=U_{B}^{\prime}\left(x, y_{s}\right) y_{s}-U_{B}\left(x, y_{s}\right), \quad b y_{s}=2 U_{B}\left(x, y_{s}\right)-U_{B}^{\prime}\left(x, y_{s}\right) y_{s} .
$$

The result is shown in figure 12. It can be seen that the composite profiles fit nicely the measurements over the whole flow height, for all the five profiles. The streamline providing the best matching (broken line) remains very close to the 


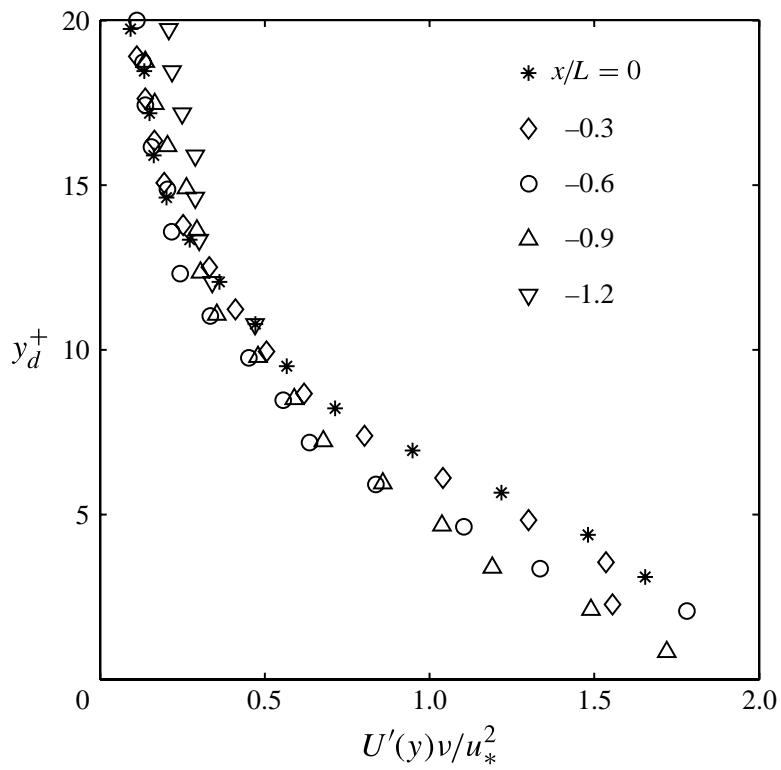

FIGURE 11. Vertical profiles of the mean velocity gradient $U^{\prime}((y)$ in the inner layer $\left(y_{d}^{+}<\delta_{i}^{+}=15\right)$, normalized with $u_{*}^{2} / \nu$, at five $x$-positions upstream of the dune crest. Parameters: see figure 3 .

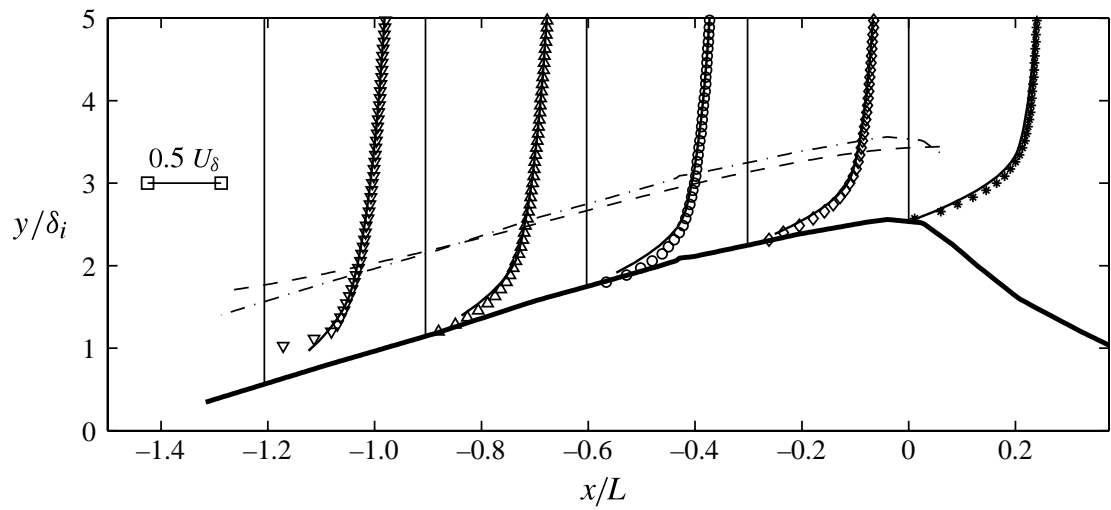

FIGURE 12. Measured velocity profiles (markers) and composite fits (solid lines): - - , streamline on which the matching of the outer Bernoulli calculation and the inner parabolic fit is performed; - - - , upper boundary of the inner layer with $\delta_{i}=1.6 \mathrm{~mm}$. Parameters: see figure 3.

line $y_{d}=\delta_{i}$ parallel to the dune profile (dash-dotted line). It is noteworthy that the 'apparent pressure gradient' $2 \mu a$ resulting from the fitting procedure is much smaller than the actual pressure gradient found at the edge of the outer layer from the Bernoulli calculation: $2 \mu a$ decreases from $-0.2 \Delta p_{\text {ref }} / L$ near the foot of the dune to $-0.6 \Delta p_{\text {ref }} / L$ at the crest, with $\Delta p_{\text {ref }}=\rho U_{\delta}^{2}(H / \delta) \approx 4.6 \mathrm{~Pa}$, whereas the actual pressure gradient is nearly uniform with value $-0.6 \Delta p_{\text {ref }} / L$. The difference between $2 \mu a$ and the actual pressure gradient corresponds to fluid acceleration, which thus represents $2 / 3$ of the pressure gradient near the dune foot, and zero near the crest. 

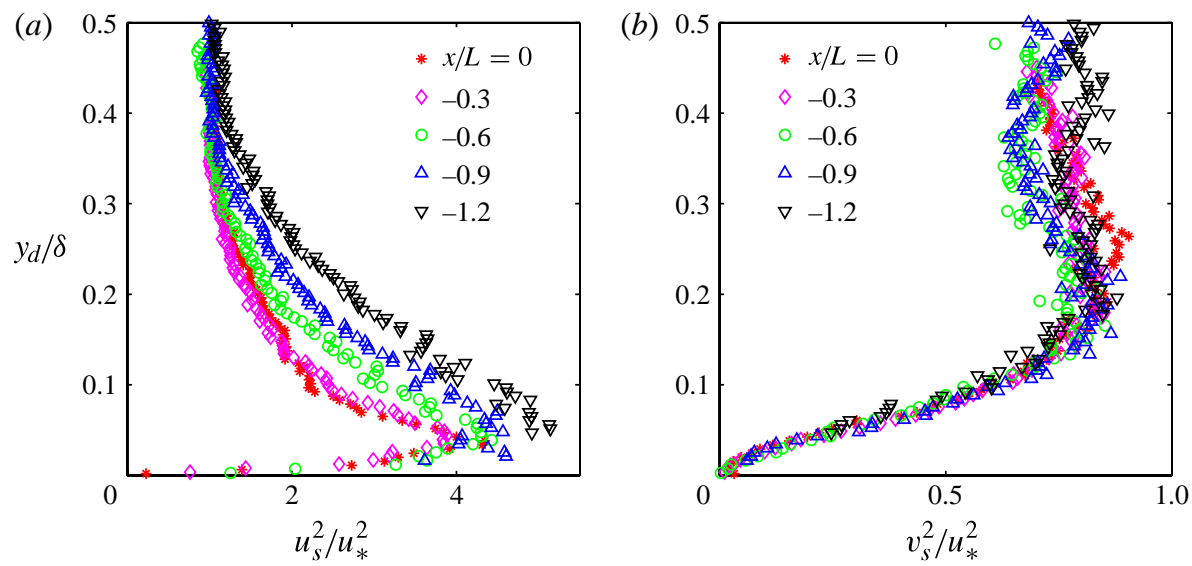

FIGURE 13. Vertical profiles of the normal Reynolds stresses, normalized with $u_{*}^{2}$, at five positions upstream of the crest: $(a) \overline{u_{s}^{2}} ;(b) \overline{v_{s}^{2}}$. Parameters: see figure 3 .

\section{Stresses}

\subsection{Reynolds stresses}

The determination of the Reynolds stresses along the dune requires the calculation of the momentum transfer across the streamlines of the mean flow. Let $u^{\prime}$ and $v^{\prime}$ be the velocity fluctuations in the Cartesian plane, and let $u_{s}$ and $v_{s}$ be the fluctuations in the curvilinear coordinate system made of the streamlines, with local angle $\alpha(x, y)$. In the curvilinear system, the transverse mean velocity $V_{s}$ is zero and the turbulent shear stress are

$$
\begin{aligned}
\overline{\left(U_{s}+u_{s}\right)\left(V_{s}+v_{s}\right)} & =\overline{u_{s} v_{s}} \\
& \left.=\overline{u^{\prime} v^{\prime}} \cos 2 \alpha-\frac{1}{2} \overline{\left(u^{\prime 2}\right.}-\overline{v^{\prime 2}}\right) \sin 2 \alpha,
\end{aligned}
$$

where the angle $\alpha(x, y)$ is obtained from $\tan \alpha=V / U$. The normal stresses $\overline{u_{s}^{2}}$ and $\overline{v_{s}^{2}}$ in the local reference frame can be computed from similar relations.

Vertical profiles of the normal Reynolds stress $\overline{u_{s}^{2}} / u_{*}^{2}$ are displayed in figure 13(a) (where $u_{*}$ is the friction velocity on the smooth wall, as before). At all the longitudinal positions, this stress increases towards the dune surface in the outer layer $\left(y_{d} \gtrsim \delta_{i}=0.05 \delta\right)$, as expected, and decreases to zero in the inner layer. Along the dune, the peak value decreases from $5 u_{*}^{2}$ to $4 u_{*}^{2}$ at the crest, while its vertical location slightly lowers, due to the compression of the streamlines. In the outer layer, $\overline{u_{s}^{2}}$ clearly decreases towards the crest with relative variation $\Delta \overline{u_{s}^{2}} / \overline{u_{s}^{2}} \approx-0.5$, or $-3 \Delta U_{L} / U_{\delta}$ in terms of the dimensionless speed-up. This decrease is, however, small in comparison with that of pressure, which, from the analysis of the previous section, is proportional to the decrease of $U^{2}$ and can be estimated as $2 U_{0}\left(\delta_{i}\right) \Delta U_{L, p e a k} \approx 70 u_{*}^{2}$.

Figure $13(b)$ displays profiles of the normal stress $\overline{v_{s}^{2}} / u_{*}^{2}$. At all the longitudinal locations, this stress increases slightly towards the dune surface, reaches a flat maximum of $0.8 u_{*}^{2}$ at $y_{d} \approx 0.2 \delta=4 \delta_{i}$, and then decreases monotonically in the inner layer. The variations along the dune are quite small, with no clear trend.

Figure 14(a) displays profiles of the Reynolds shear stress $-\overline{u_{s} v_{s}}$. These profiles exhibit a smooth peak at the location $y_{d} \approx 0.15 \delta=3 \delta_{i}$ and decrease monotonically towards the dune surface in the inner layer. The variation along the dune of the peak 

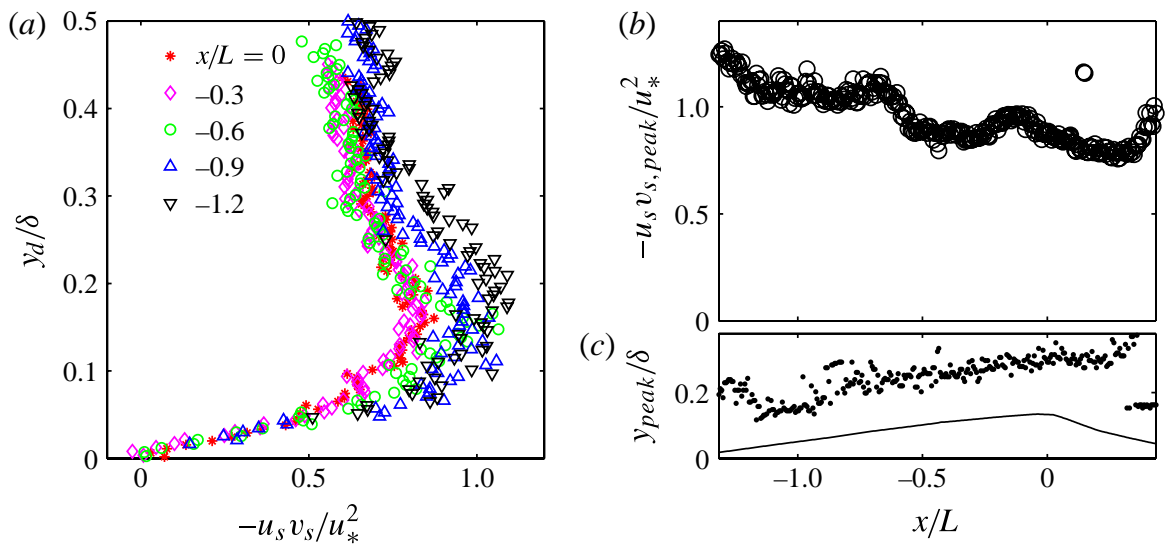

FIGURE 14. (a) Vertical profiles of the Reynolds shear stress $-\overline{u_{s} v_{s}}$, normalized with $u_{*}^{2}$, at five positions upstream of the crest. (b) Variation along the dune of the value of the peak. $(c)$ Variation along the dune of the vertical location of the peak. Parameters: see figure 3.

value and its vertical location are plotted in figures $14(b)$ and 14(c); although the measurements exhibit some scatter, the peak value clearly decreases by about $25 \%$, from $1.1 u_{*}^{2}$ near the dune foot to $0.8 u_{*}^{2}$ at the crest, approximately.

These results agree with previous measurements over isolated hills or wavy surfaces (Buckles, Hanratty \& Adrian 1984; Ross et al. 2004), and with numerical simulations for similar Reynolds numbers (Gong et al. 1996; De Angelis et al. 1997; Henn \& Sykes 1999; Yue et al. 2006). They are also consistent with the rapid distortion theory which predicts a decrease of $\overline{u_{s}^{2}}$ in regions of accelerated flow, and no significant variation of $\overline{v_{s}^{2}}$ for the typical anisotropy ratio $\overline{u_{s}^{2}} / \overline{v_{s}^{2}} \approx 3$ measured at $y_{d}=0.2 \delta \approx 4 \delta_{i}$ (Britter et al. 1981; Finnigan et al. 1990; Ross et al. 2004).

It can be concluded that the longitudinal gradient of the Reynolds stresses is small in the whole flow, in comparison with that of pressure. The vertical gradient of the shear stress is significant in the inner layer only, with magnitude $-\partial_{y} \overline{u_{s} v_{s}} \approx u_{*}^{2} / \delta_{i} \approx 14 u_{*}^{2} / L$, comparable to that of the horizontal pressure gradient, $\partial_{x} p / \rho \approx 70 u_{*}^{2} / L$. The difference between these gradients results in fluid acceleration in the inner layer. The pressure measurements and momentum budgets presented by Finnigan et al. (1990), for wind tunnel experiments over a rough ridge, led to similar conclusions.

\subsection{Mixing length}

A crucial assumption of most theoretical analyses is that, within the inner layer, the Reynolds shear stress can be modelled using the concept of mixing length, defined as

$$
-\overline{u_{s} v_{s}}=\ell^{2}\left(\frac{\mathrm{d} U}{\mathrm{~d} y}\right)^{2},
$$

with the standard model $\ell=\kappa y_{d}$, or more elaborated models accounting for the effects of shear and 'blocking' of the normal component of turbulence (Weng et al. 1991; Belcher \& Hunt 1998; Ross et al. 2004). This assumption can be assessed from the determination of $\ell$ from the above relation and the measured Reynolds stress and mean velocity gradient. Figure 15 displays vertical profiles of the mixing length thus determined. For $y_{d} \lesssim 0.5 \delta_{i} \approx \delta_{v}$ where $\delta_{v}=7 v / u_{*}$ is the thickness of the viscous 


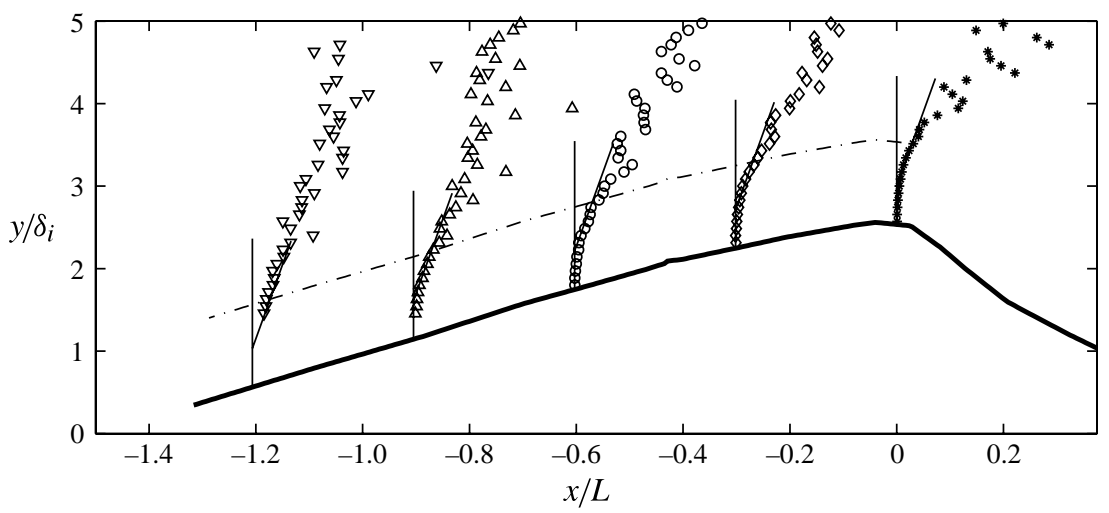

FIGURE 15. Vertical profiles of the mixing length $\ell$ determined from (4.2), with the measured Reynolds stress and mean velocity gradient:,$- \ell=\kappa\left(y_{d}-\delta_{v}\right)$ with $\delta_{v}=7 v / u_{*}$; $-\cdot-$, upper limit of the inner layer $y_{d}=\delta_{i}$. Parameters: see figure 3.

sublayer, the mixing length is nearly zero, as expected. For $y_{d} \gtrsim \delta_{v}$, it increases linearly with the standard slope $\kappa$ (the solid lines correspond to $\ell=\kappa\left(y_{d}-\delta_{v}\right)$ ), and still increases in the outer layer according to the same law, approximately. Finally, the standard mixing length here accounts for the vertical variation of the Reynolds shear stress above the upstream face of the dune, provided that the 'blocking' caused by the viscous sublayer is accounted for (Hunt et al. 2006).

\subsection{Total shear stress}

The total shear stress along a streamline is the sum of the Reynolds and viscous stresses

$$
-\rho \overline{u_{s} v_{s}}+\mu \frac{\partial U}{\partial y},
$$

where the contribution of the omitted terms, of second order in the small slope of the streamlines, are negligible, as we have verified. Figure 16(a) displays vertical profiles of this shear stress. In the outer layer and the upper half of the inner layer $\left(y_{d} \gtrsim 0.5 \delta_{i} \approx \delta_{v}\right)$, the shear stress decreases slightly as the Reynolds stress, displayed in figure 14(a), since viscous stresses are negligible. Within the viscous sublayer, $y_{d} \lesssim \delta_{v}$, the total stress increases strongly, approximately linearly: whereas the turbulent stress vanishes, the viscous stress becomes large as the mean velocity gradient does (see figure 11). The resulting change of curvature of the stress profile corresponds to that previously observed, for the Reynolds stresses, for flows over rough surfaces (Finnigan et al. 1990; Weng et al. 1991; Athanassiadou \& Castro 2001; Ross et al. 2004). The increase here occurs within the viscous sublayer since it is a large part of the inner layer.

The actual shear stress $\tau_{d}$ acting at the dune surface can be obtained from the extrapolation of the profiles of the total shear stress. As shown in figure 16(b) (circles), it appears that $\tau_{d}$ increases approximately linearly along the dune, and reaches its maximum value, about $2 \rho u_{*}^{2}$, at the dune crest. Beyond the crest, the shear stress strongly decreases, as expected. It is noteworthy that the maximum shear stress is reached at the dune brink, whereas theoretical linear analyses predict that it is located upstream of the crest of a gentle bump (Benjamin 1959; Hunt et al. 1988). Nonlinear effects might be invoked here, or the fact that the dune profile is not smooth but 

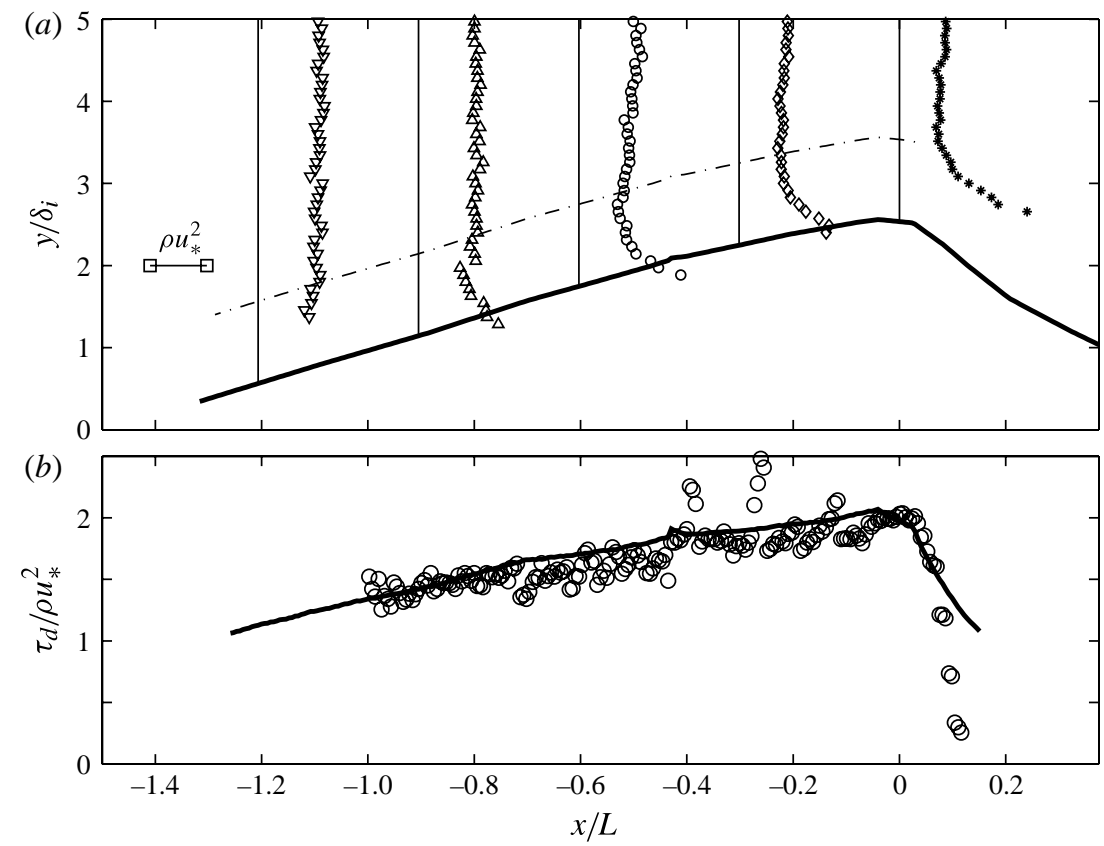

FIGURE 16. (a) Vertical profiles of the shear stress (turbulent and viscous) above the dune: $-\cdot-$, upper limit of the inner layer. $(b) \circ$, measured shear stress extrapolated down to the dune surface; - , shear stress from the parabolic fit of the mean velocity profile with coefficients given by (3.6). Parameters: see figure 3 .

exhibits a sharp brink with positive slope (see $\$ 2.3$ ). Figure $16(b)$ also displays the shear stress derived from the parabolic fit of the inner velocity (solid line), i.e. $\mu b$ with $b$ defined from (3.6). The agreement with the measurements is remarkable. The streamline chosen for the matching, very close to the inner layer boundary $y_{d}=\delta_{i}$ (see figure 12), provides a good fit over the whole dune profile. Finally, large shear stress variations do occur normal to the dune surface, but these variations take place in the viscous sublayer, in agreement with a conjecture by Sykes (1980). Along the dune, the increase by a factor of two of the surface shear stress is similar to that found for large aeolian dunes, from rough estimates assuming logarithmic velocity profiles close to the ground (Weng et al. 1991; Kroy et al. 2002).

\section{Variations with the flow velocity}

All the results discussed above are for Reynolds number $R e=9300$, for which the shear stress on the dune was below the threshold for particle motion, so that the dune was at rest. Similar measurements have been performed for three Reynolds numbers above the threshold, i.e. for moving dunes, namely $R e=14300,16200$ and 18300 , and corresponding inner layer thickness $\delta_{i}=1.6,1.5$ and $1.4 \mathrm{~mm}$. These measurements showed essentially the same results. Three figures displaying the measured Lagrangian speed-up $\Delta U_{L}$, similar to figure 8 , are available as supplementary material at journals. cambridge.org/flm. In all cases, the peak of the speed-up lies near the top of the inner layer, and (3.5) provides a good representation of the flow in the outer layer.

The variation along the dune of the peaks of the speed-up and vertical velocity is shown in figure 17. The maximum speed-up always occurs at the dune brink 

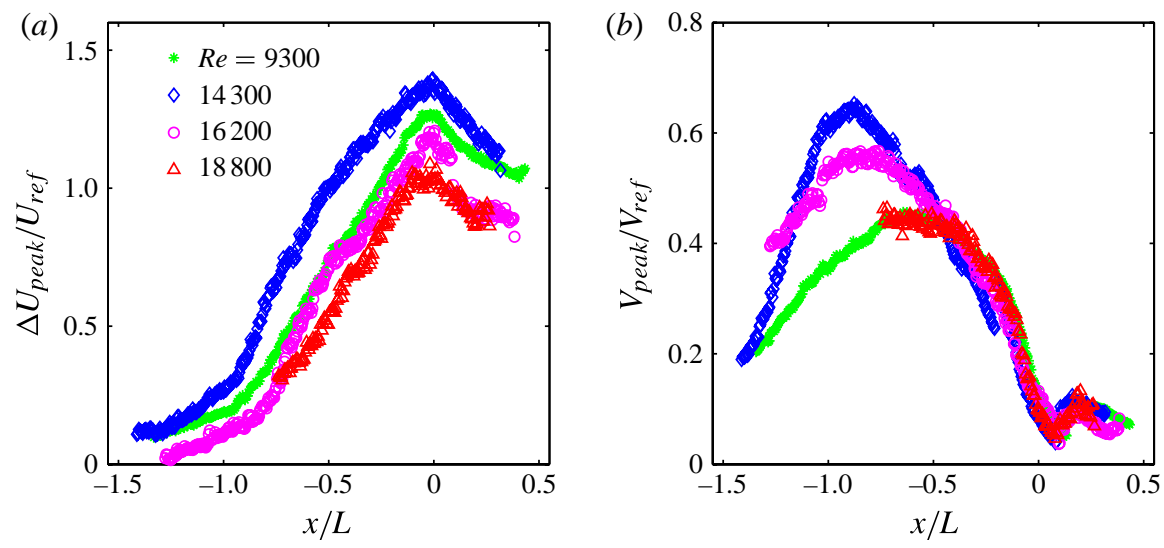

FIGURE 17. Variation along the dune, for four Reynolds numbers, of (a) the peak $\Delta U_{L, p e a k}$ of the speed-up normalized by $U_{r e f}=(H / \delta) U_{\delta}$, and $(b)$ the peak $V_{\text {peak }}$ of the vertical mean velocity normalized by $V_{r e f}=(H / L) U_{\delta}$. The dune lengths and heights are as follows: $R e=9260, L=23 \mathrm{~mm}$ and $H=4.0 \mathrm{~mm} ; R e=14300, L=28 \mathrm{~mm}$ and $H=3.6 \mathrm{~mm}$; $R e=16200, L=27 \mathrm{~mm}$ and $H=3.6 \mathrm{~mm} ; R e=18800, L=22 \mathrm{~mm}$ and $H=3.6 \mathrm{~mm}$.

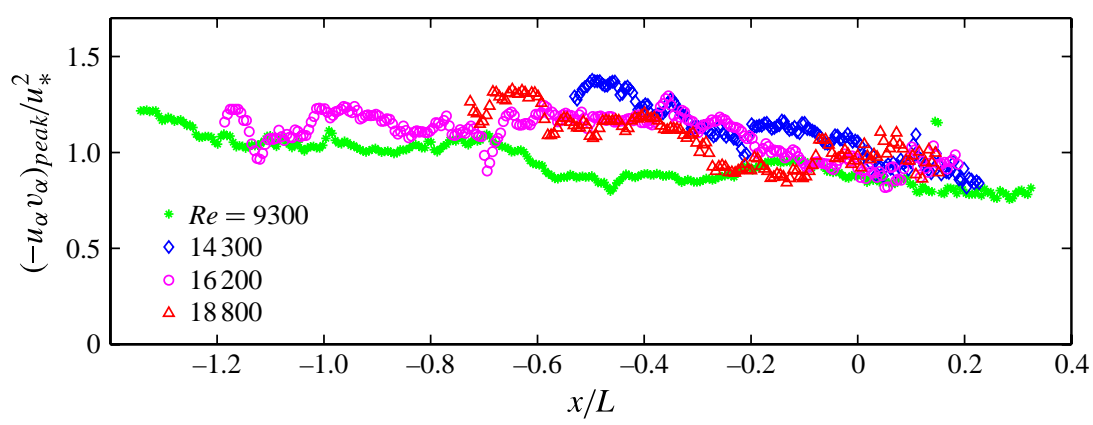

FIGURE 18. Variation along the dune of the peak of the normalized turbulent shear stress $-\overline{u_{s} v_{s}}$, for four Reynolds numbers and the same dunes as in figure 17.

(figure $17 a$ ) with values in the range $1-1.4 U_{\text {ref }}$, where $U_{r e f}=(H / \delta) U_{\delta}$ is the expected order of magnitude. Except for $R e=9300$ (where the dune is at rest), the maximum speed-up decreases with increasing Reynolds number. Using for the speed-up the scale $\left(U_{\delta} / U_{0}\left(\delta_{i}\right)\right)^{2}(H / L) U_{\delta}$ (Belcher \& Hunt 1998), rather than $(H / \delta) U_{\delta}$, gives close results. The vertical velocity profiles shown in figure $17(b)$ display a maximum in the range 0.45-0.65, which takes place approximately at the middle of the upstream face.

The profiles of the turbulent stresses and mixing length (not shown) are very similar to those presented in the previous section for $R e=9300$. Variations along the dune of the maximum Reynolds shear stress (peak value) are displayed in figure 18. The measurements exhibited some scatter, which may be due to the slow motion of the dune or three-dimensional flow structures related to the lateral meandering of the streaks observed at the dune surface (see Part 1). A running average over a few points has been performed in order to make the variations more visible. For all Reynolds numbers, the peak of the turbulent shear stress can be seen to decrease slightly towards the crest, from $1.3 u_{*}^{2}$ to $0.9 u_{*}^{2}$ approximately. 


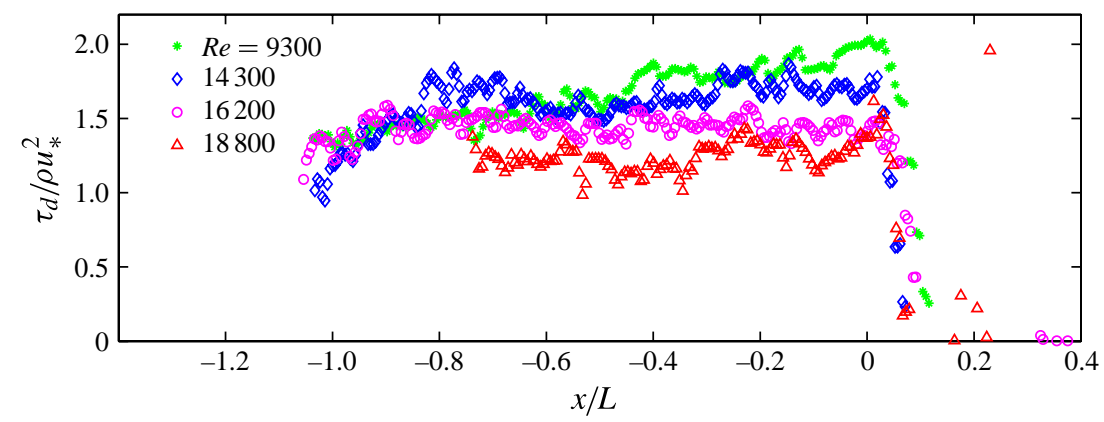

FIGURE 19. Variation along the dune of the normalized shear stress $\tau_{d} / \rho u_{*}^{2}$ at the dune surface, for four Reynolds numbers and the same dunes as in figure 17.

Finally, figure 19 displays the normalized shear stress at the dune surface, obtained as in figure $16(b)$ from extrapolation. For the smallest Reynolds number, the shear stress increases towards the crest, as noted in the previous section (dune at rest). For the three larger Reynolds number (moving dunes), the increase is less pronounced. At the brink, the normalized shear stress clearly decreases as the Reynolds number increases, from $2.0 \rho u_{*}^{2}$ for $R e=9300$ down to $1.5 \rho u_{*}^{2}$ for $R e=18800$. The reason for this decrease may be related to a Reynolds number effect, or a relaxation of the fluid flow induced by the grain motion. Further investigations are needed to clarify this point.

\section{Summary and discussion}

Measurements have been presented of the flow over erodible barchan dunes, below and above the threshold for particle motion, for moderate Reynolds numbers and hydrodynamically smooth regimes. Particular attention has been paid to the flow over the upstream face of the dune where the particles are entrained, down to $y_{d}^{+} \approx 1$ so that the flow in the viscous sublayer was fully resolved. The value of $H / L$ was sufficiently small, and that of $\ln \left(L / y_{0}\right)$ sufficiently large, for the measurements to be compared to linear asymptotic analyses. The results can be summarized as follows.

(a) The fluid flow accelerates towards the dune crest, and a peak develops in the vertical profiles of the speed-up, located near the inner layer boundary, at $y_{d} \approx \delta_{i}$. The Lagrangian speed-up, i.e. the actual velocity variation of the fluid particles, is significantly smaller, by $50 \%$, than the 'same-elevation' speed-up generally considered. The maximum speed-up, of about $(H / \delta) U_{\delta}$, is reached at the dune brink, with normalized value decreasing slightly as the Reynolds number is doubled.

(b) The perturbed flow has the classical two-layer structure: an inviscid outer layer and a viscous (turbulent) inner layer with thickness $\delta_{i}$. In the outer layer, the effect of streamline curvature is of the same order of magnitude as the Bernoulli effect related to the compression of the streamlines: streamline curvature inhibits the speed-up near the foot of the dune and enhances it near the crest; in other words, ignoring the transverse pressure gradient (as in the boundary layer equations or the Saint-Venant equations widely used in engineering) leads to a large underestimate, by a factor of two, of the speed-up and surface shear stress.

(c) In the inner layer, the analysis of Hunt et al. (1988) fails to predict the right flow, as expected because of the large thickness of the viscous sublayer $\left(\delta_{v} \approx 0.5 \delta_{i}\right)$. 
Alternatively, a parabolic fit of the velocity profiles, matched with the outer velocity at $y_{d} \approx \delta_{i}$, provides a good description of the entire flow. Near the upstream foot of the dune, the pressure gradient inherited from the outer layer is balanced two-thirds by the fluid acceleration and one-third by the shear stress; near the crest, fluid acceleration vanishes and shear stress dominates.

(d) Reynolds stresses have been determined in the reference frame of the streamlines. Their vertical profiles exhibit a well-defined peak, located in the lower part of the outer layer. The peak values of $\overline{u_{s}^{2}}$ and $-\overline{u_{s} v_{s}}$ decrease slightly towards the crest, whereas that of $\overline{v_{s}^{2}}$ remains constant, in qualitative agreement with the rapid distortion theory. In the inner layer, the shear stress $-\overline{u_{s} v_{s}}$ decreases monotonically towards the smooth dune surface. The standard mixing length model was shown to be relevant, up to $y_{d} \approx 2 \delta_{i}$, provided the blocking effect of the viscous sublayer is accounted for, i.e. $\ell=\kappa\left(y_{d}-\delta_{v}\right)$. The gradients of the Reynolds stresses are negligible except for the vertical gradient of the shear stress which, in the inner layer, is $1 / 5$ of the longitudinal pressure gradient. The total stress, viscous and turbulent, increases strongly across the viscous sublayer, in agreement with a conjecture by Sykes (1980). This increase corresponds to that of the Reynolds shear stress over hydrodynamically rough hills or dunes (Finnigan et al. 1990; Weng et al. 1991).

(e) At the dune surface, the total shear stress increases towards the crest where it reaches $2 \rho u_{*}^{2}$, i.e., twice its unperturbed value. This value is similar to that predicted for rough surfaces, or measured (assuming logarithmic profiles) over large aeolian dunes (Weng et al. 1991; Sauermann et al. 2003). The maximum shear stress is reached at the brink, unlike the case of a gentle bump, where the maximum is reached upstream of the summit, indicating that the slip face and flow separation play an important role in the distribution of shear stress (consistent with the theory of boundary-layer separation). Thus, flow calculations replacing the dune and the recirculation bubble by a smooth envelope must be considered with caution, as noted by Kroy et al. (2002); three-dimensional effects, not investigated here, might also be of importance.

( $f$ ) As the Reynolds number is increased and the dune moves, the above features remain essentially the same. However, weak dependences with $R e$ were found: doubling $R e$, the normalized maximum speed-up and vertical velocity decrease by $30 \%$, and the normalized surface shear stress decreases by $30 \%$ too. This decrease might be related to the decreasing importance of viscosity or the increasing particle motion; further experiments are needed to clarify this point.

Finally, the investigation of the water flow over a small isolated sand dune allows detailed assessment of theoretical analyses. Some important questions, however, remain unanswered, such as the structure of the flow near the horns (which controls the small particle leak), the relationship between the surface shear stress and the particle flux (which controls the dune velocity and stability), or the effect of the fluid streaks reported in Part 1 of this study. These questions are left for future work.

\section{Acknowledgements}

We thank J. Hinch and P. Luchini for stimulating discussions and helpful suggestions. We also thank S. Cazin for valuable technical help. We are grateful to the French Agence Nationale de la Recherche for partial financial support for 
this study (no. ANR-07-BLAN-0180-01), and to the Brazilian government foundation CAPES for E.M.F.'s scholarship grant.

Supplementary material is available at journals.cambridge.org/flm.

\section{Appendix. Flow in the inner layer, after Hunt et al. (1988)}

In this appendix we summarize the asymptotic analysis of Hunt et al. (1988) and discuss its application to channel flow. Consider the turbulent flow over a dune of length $L$ and height $H \ll L$, with profile $h(x)=H f(x / L)$ and roughness $y_{0}$. Far upstream of the dune, the unperturbed velocity $U_{0}(y)$ is logarithmic in the boundary layer of thickness $\delta_{B L}$. Above the dune, the flow disturbance can be considered as the superposition of an inner layer where the standard mixing length theory is assumed to be relevant, and an essentially inviscid outer layer. The thickness $\delta_{i}$ of the inner layer is defined by $\left(\delta_{i} / L\right) \ln \left(\delta_{i} / y_{0}\right)=2 \kappa^{2}$.

The outer layer itself is composed of an upper irrotational layer, and a middle rotational layer where the vertical pressure gradient $\partial_{y} p$ is assumed to be negligible. For long hills, i.e. $L>\delta_{B L}$, the boundary $\delta_{m}$ between the upper and middle layers is located at the displaced height $\delta_{m}=\delta_{B L}$ above the hill; for shorter hills, $\delta_{m}=L \ln ^{-1 / 2}\left(L / y_{0}\right)$. The solution in the outer layer provides in particular the pressure $P(x)$ which drives the flow in the inner layer.

In the inner layer, the solution of the linearized problem can be sought as a power series in the parameter $\ln ^{-1}\left(\delta_{i} / y_{0}\right) \ll 1$, such that, for the longitudinal velocity perturbation,

$$
\frac{u_{d}}{U_{0}\left(\delta_{m}\right)}=\frac{H / L}{U_{0}\left(\delta_{i}\right) / U_{0}\left(\delta_{m}\right)}\left(u_{d}^{(0)}(X, \zeta)+\ln ^{-1}\left(\delta_{i} / y_{0}\right) u_{d}^{(1)}(X, \zeta)\right),
$$

where $X=x / L, \zeta=(y-h(x)) / \delta_{i}$ is the normalized vertical distance above the dune, and $U_{0}(y)=\left(u_{*} / \kappa\right) \ln \left(y / y_{0}\right)$. Taking the Fourier transform in $X$, e.g.

$$
u_{d}^{(0)}(X, \zeta)=\frac{1}{2 \pi} \int_{-\infty}^{\infty} \hat{u}_{d}^{(0)}(k, \zeta) \mathrm{e}^{\mathrm{i} k x} \mathrm{~d} k
$$

the solution for the Fourier components of $u_{d}$ is found to be

$$
\begin{gathered}
\hat{u}_{d}^{(0)}=\hat{\sigma}(k) \\
\hat{u}_{d}^{(1)}=\hat{\sigma}(k)\left(1-\log \zeta-4 \mathrm{~K}_{0}\left[2(\mathrm{i} k \zeta)^{1 / 2}\right]\right),
\end{gathered}
$$

where $\mathrm{K}_{0}$ is a modified Bessel function and $-\hat{\sigma}(k)=-k \hat{f}(k)$ is the Fourier transform of the pressure perturbation inherited from the outer layer and normalized with $\rho U_{0}^{2}\left(\delta_{m}\right)$. For the bell-shaped dune $f(X)=\left(1+X^{2}\right)^{-1}$, the Fourier transform is $\hat{f}(k)=\pi \mathrm{e}^{-|k|}$, and for the Gaussian dune $f(X)=\mathrm{e}^{-X^{2}}$, it is $\hat{f}(k)=\sqrt{\pi} \mathrm{e}^{-k^{2} / 4}$.

The shear stress on the dune is given by

$$
\frac{\tau_{d}}{\rho U_{0}^{2}\left(\delta_{m}\right)}=2 \frac{H / L}{\left(U_{0}\left(\delta_{i}\right) / U_{0}\left(\delta_{m}\right)\right)^{2}}\left(\tau_{d}^{(0)}(X, \zeta)+\ln ^{-1}\left(\delta_{i} / y_{0}\right) \tau_{d}^{(1)}(X, \zeta)\right),
$$

with

$$
\begin{gathered}
\hat{\tau}_{d}^{(0)}=\hat{\sigma}(k), \\
\hat{\tau}_{d}^{(1)}=\hat{\sigma}(k)(2 \ln k+4 \gamma+1+\mathrm{i} \pi),
\end{gathered}
$$

where $\gamma \approx 0.5772$ is the Euler constant. 
Given that the half-height $\delta$ of the channel represents some boundary layer thickness and that the dunes are 'long' in the sense that $L>\delta$, we chose $U_{0}\left(\delta_{m}\right)=U_{\delta}$ (the centreline velocity), consistent with the analysis of Hunt et al. (1988) - hence the velocity profiles shown in figure 12. A different choice for $U_{0}\left(\delta_{m}\right)$ would lead to minor changes. A more accurate asymptotic theory for the pressure-driven flow above dunes in closed channels or pipes remains to be performed.

\section{REFERENCES}

Abrams, J. \& HANRATTy, T. J. 1985 Relaxation effects observed for turbulent flow over a wavy surface. J. Fluid Mech. 151, 443-455.

Andreotti, B., Claudin, P. H. \& Douady, S. 2002 Selection of dune shapes and velocities. Part 2. A two-dimensional modelling. Eur. Phys. J. B 28, 341-352.

Athanassiadou, M. \& CAstro, I. P. 2001 Neutral flow over a series of rough hills: a laboratory experiment. Boundary-Layer Meteorol. 101, 1-30.

Belcher, S. E. \& Hunt, J. C. R. 1998 Turbulent flow over hills and waves. Annu. Rev. Fluid Mech. 30, 507-538.

Benjamin, T. B. 1959 Shearing flow over a wavy boundary. J. Fluid Mech. 6, 161-205.

BEST, J. 2005 The fluid dynamics of river dunes: a review and some future research directions. J. Geophys. Res. 110, F04S02.

van Boxel, J. H., Arens, S. M. \& VAn DiJK, P. M. 1999 Aeolian processes across transverse dunes. Part I. Modelling the air flow. Earth Surf. Process. Landf. 24, 255-270.

Britter, R. E., Hunt, J. C. R. \& Richards, K. J. 1981 Air flow over a two-dimensional hill: studies of velocity speed-up, roughness effects and turbulence. Q. J. R. Meteorol. Soc. 107, 91-110.

Buckles, J., HanRatty, T. J. \& Adrian, R. J. 1984 Turbulent flow over large-amplitude wavy surfaces. J. Fluid Mech. 140, 27-44.

Colombini, M. \& Stocchino, A. 2008 Finite-amplitude river dunes. J. Fluid Mech. 611, 283-306.

Colombini, M. \& Stocchino, A. 2011 Ripple and dune formation in rivers. J. Fluid Mech. 673, $121-131$.

DAVIDSON, P. A. 2004 Turbulence: An Introduction for Scientists and Engineers. Oxford University Press.

De Angelis, V., Lombardi, P. \& BanerJee, S. 1997 Direct numerical simulation of turbulent flow over a wavy wall. Phys. Fluids 9, 2429-2442.

Finnigan, J. J. \& Belcher, S. E. 2004 Flow over a hill covered with a plant canopy. $Q$. J. $R$. Meteorol. Soc. 130, 1-29.

Finnigan, J. J., Raupach, M. R., Bradley, E. F. \& Aldis, G. K. 1990 A wind tunnel study of turbulent flow over a two-dimensional ridge. Boundary-Layer Meteorol. 50, 277-317.

Fourrière, A., Claudin, P. \& Andreotti, B. 2010 Bedforms in a turbulent stream: formation of ripples by primary linear instability and of dunes by nonlinear pattern coarsening. J. Fluid Mech. 649, 287-328.

Franklin, E. M. \& ChARRU, F. 2011 Subaqueous barchan dunes in turbulent shear flow. Part 1. Dune motion. J. Fluid Mech. 675, 199-222.

Gong, W. \& IbBetson, A. 1989 A wind tunnel study of turbulent flow over model hills. Boundary-Layer Meteorol. 49, 113-148.

Gong, W., TAYlor, P. A. \& Dörnbrack, A. 1996 Turbulent boundary-layer flow over fixed aerodynamically rough two-dimensional sinusoidal waves. J. Fluid Mech. 312, 1-37.

HenN, D. S. \& SYKES, R. I. 1999 Large-eddy simulation of flow over wavy surfaces. J. Fluid Mech. 383, 75-112.

Hunt, J. C. R., Eames, I. \& Westerweel, J. 2006 Mechanics of inhomogeneous turbulence and interfacial layers. J. Fluid Mech. 554, 499-519.

Hunt, J. C. R., Leibovich, S. \& Richards, K. J. 1988 Turbulent shear flows over low hills. Q. J. R. Meteorol. Soc. 114, 1435-1470. 
JACKson, P. S. \& HUnt, J. C. R. 1975 Turbulent wind flow over a low hill. Q. J. R. Meteorol. Soc. 101, 929-955.

Kostaschuk, R., Villard, P. \& Best, J. 2004 Measuring velocity and shear stress over dunes with acoustic Doppler profiler. J. Hydraul. Engng ASCE 130, 932-936.

Kroy, K., Sauermann, G. \& Herrmann, H. J. 2002 Minimal model for aeolian sand dunes. Phys. Rev. E 66, 031302.

Livingstone, I., Wiggs, G. F. S. \& Weaver, C. M. 2007 Geomorphology of desert sand dunes: a review of recent progress. Earth-Sci. Rev. 80, 239-257.

MAson, P. J. \& SYKeS, R. I. 1979 Flow over an isolated hill of moderate slope. Q. J. R. Meteorol. Soc. 105, 383-395.

Panton, R. L. 2007 Composite asymptotic expansions and scaling wall turbulence. Phil. Trans. R. Soc. Lond. A 365, 733-754.

Poggi, D., Katul, G. G., Albertson, J. D. \& Ridolfi, L. 2007 An experimental investigation of turbulent flow over a hilly surface. Phys. Fluids 19, 036601.

RICHARDS, K. J. 1980 The formation of ripples and dunes on an erodible bed. J. Fluid Mech. 99, 597-618.

Ross, A. N., Arnold, S., Vosper, S. B., Mobbs, S., Dixon, N. \& Robins, A. G. 2004 A comparison of wind-tunnel experiments and numerical simulations of neutral and stratified flow over a hill. Boundary-Layer Meteorol. 113, 427-459.

Sauermann, G., Andrade, J. S., Maia, L. P., Costa, U. M. S., Araújo, A. D. \& HERRMANN, H. J. 2003 Wind velocity and sand transport on a barchan dune. Geomorphology 54, 245-255.

Scheichl, B., Kluwick, A. \& SMith, F. T. 2011 Break-away separation for high turbulence intensity and large Reynolds number. J. Fluid Mech. 670, 260-300.

Schlichting, H. \& Gesten, K. 2000 Boundary-Layer Theory. Springer.

Smith, J. D. \& MCLEAN, S. R. 1977 Spatially averaged flow over a wavy surface. J. Geophys. Res. 82, 1735-1746.

SUMER, B. \& BAKIOGLU, M. 1984 On the formation of ripples on an erodible bed. J. Fluid Mech. 144, 177-190.

SYKES, R. I. 1980 An asymptotic theory of incompressible turbulent boundary-layer flow over a small hump. J. Fluid Mech. 101, 647-670.

TAylor, P. A., Mason, P. J. \& Bradley, E. F. 1987 Boundary-layer flow over low hills. A review. Boundary-Layer Meteorol. 39, 107-132.

Teunissen, H. W., Shokr, M. E., Bowen, A. J., Wood, C. J. \& Green, D. W. R. 1987 The Askervein Hill project: wind-tunnel simulations at three length scales. Boundary-Layer Meteorol. 40, 1-29.

WALKeR, I. J. \& NickLING, W. G. 2003 Simulation and measurement of surface shear stress over isolated and closely spaced transverse dunes in a wind tunnel. Earth Surf. Process. Landf. 28, $1111-1124$.

WENG, W. 1997 Stably stratified boundary-layer flow over low hills: a comparison of model results and field data. Boundary-Layer Meteorol. 85, 223-241.

Weng, W. S., Hunt, J. C. R., Carruthers, D. J., Warren, A., Wiggs, G. F. S., Livingstone, I. \& CASTRO, I. 1991 Air flow and sand transport over sand-dunes. Acta Mechanica Suppl. 2, 1-22.

Wiggs, G. F. S., Livingstone, I. \& WARREN, A. 1996 The role of streamline curvature in sand dune dynamics: evidence from field and wind tunnel measurements. Geomorphology 17, $29-46$.

Yue, W., Lin, C.-L. \& PAtel, V. C. 2006 Large-eddy simulation of turbulent flow over a fixed two-dimensional dune. J. Hydraul. Engng ASCE 132, 643-651.

ZEMAN, O. \& JENSEN, N. O. 1987 Modification of turbulence characteristics in flow over hills. Q. J. R. Meteorol. Soc. 113, 55-80. 